\title{
Semianalytical modeling of multistage bunch compression with collective effects
}

\author{
Igor Zagorodnov* and Martin Dohlus \\ Deutsches Elektronen-Synchrotron (DESY), Notkestrasse 85, 22603 Hamburg, Germany
}

(Received 6 July 2010; published 13 January 2011)

\begin{abstract}
In this paper we introduce, for the first time, an analytical solution (up to the third order) for a multistage bunch compression and acceleration system without collective effects. The solution for the system with collective effects is found by an iterative procedure based on this analytical result. The developed formalism is applied to the two-stage Free Electron Laser in Hamburg (FLASH) and to the three-stage European XFEL. Analytical estimations of rf tolerances are given and a simple formula for the optimal compression factor in the first bunch compressor is derived.
\end{abstract}

DOI: 10.1103/PhysRevSTAB.14.014403

PACS numbers: 29.27.Bd, 41.60.Cr

\section{INTRODUCTION}

Free-electron lasers require an electron beam with high peak current and low transverse emittance. In order to meet these requirements several bunch compressors are usually planned in the beam line [1-7] to reach an extreme final compression. For example, in several projects (the European XFEL [4], FACET [5], NLC [6], ILC [7]) three-stage bunch compression and acceleration schemes will be used. The nonlinearities of the radio frequency (rf) fields and of the bunch compressors (BC's) distort the longitudinal phase space. As a result, very sharp spikes in current profile can be generated, which may drive unwanted collective effects such as coherent synchrotron radiation (CSR) and wakefields [8].

The nonlinearities of rf fields and of BC's can be corrected with a higher harmonic rf system [8-12]. A suggestion to use rf field at harmonics of the fundamental frequency 'to 'flattop' the dependence of the energy gain vs rf phase by canceling the second derivative of the fundamental at its peak " was done in [9]. A more advanced treatment with a correction of the second-order effects in a one-stage bunch compression system was presented in $[8,10]$. An analytical solution for cancellation of $\mathrm{rf}$ and BC's nonlinearities up to the third-order terms for one-stage bunch compression system was developed in [11]. The second-order treatment of multistage bunch compression systems was done in [12], where the difficulty to extend the third-order analysis to multistage systems was pointed out as well. Modeling of a two-stage bunch compression with wakefields was considered in [13], where the working points were found by "trial and error" with numerical codes.

In this paper we present, for the first time, an analytical solution for the nonlinearity correction up to the third order in a multistage bunch compression and acceleration system without collective effects for an arbitrary number of

\footnotetext{
*Corresponding author. igor.zagorodnov@desy.de
}

stages. Under the analytical solution we mean the equations which convert the parameters of the desired compression scenario in the technical values of $\mathrm{rf}$ voltages and phases to be used in the accelerating modules. In this work we follow a little different point of view on the usage of the high harmonic rf system. An installation of the higher harmonic module in a multistage bunch compression and acceleration system gives us two additional degrees of freedom for the longitudinal phase space manipulation. With these two additional rf parameters we are able to control the first and the second derivative of the global compression curve at the position of the reference particle. In other words we do not require cancellation of the higher order effects. Instead of this we impose a desired behavior of the global compression at the reference point. Let us note that even for the one-stage system our solution is much simpler than that presented in [11].

A more general solution for a system with collective effects (space-charge forces, wakefields, CSR within the chicane magnets) is found by an iterative tracking procedure based on this analytical result. The developed iterative procedure uses the analytical solution as a starting point and as a "preconditioner" for efficient correction of the $\mathrm{rf}$ parameters. It shows a fast convergence and allows for a relatively fast finding of the working points. We have applied the developed formalism to study the two-stage bunch compression scheme at FLASH [1] and the three-stage bunch compression scheme at the European XFEL [4]. The analytical estimations of the rf tolerances are given in the paper for two- and three-stage bunch compression in the explicit form and a simple formula for the optimal compression factor in the first bunch compressor is derived.

Finally, we would like to note that the longitudinal wakefields are relatively weak in the considered facilities and the working points with collective effects included are relatively close to the analytical solutions. In order to show that our formalism works even for the case of strong wakefields (for example, in projects [2,3]), we have done a test simulation of the FLASH facility with artificially increased very strong wakefields. 


\section{ANALYTICAL SOLUTION OF MULTISTAGE BUNCH COMPRESSION PROBLEM WITHOUT COLLECTIVE EFFECTS}

In order to produce an intense low emittance beam we have to start with a relatively long bunch from the gun (because of the strong space-charge forces at low energy) and to compress it later extremely. Preserving of the low emittance requires the final compression to be done only at high energy of the beam. In the case of the one-stage bunch compression scheme, the linearization of the longitudinal phase space requires a very high voltage of the high harmonic rf module. From the other side this rf voltage is decelerating and an additional $\mathrm{rf}$ power in the fundamental harmonic is required to compensate the loss in the beam energy. In order to avoid it, the correction of the longitudinal phase space is reasonable to carry out at low energy. It is possible only with the usage of the multistage bunch compression scheme. Most of the projects use a two-stage bunch compression scheme. The European XFEL [4], FACET [5], and NLC [6] will use three-stage bunch compression schemes. The exploitation of more stages of compression can be planned in the future projects as well.

At the first stage of the design only nonlinearities in $\mathrm{rf}$ fields and in BC's can be considered. But an analytical solution to convert the macroscopic description of the desired compression scheme in rf parameters of the accelerating modules is available in the literature only for the one-stage bunch compression scheme [11]. In the following sections we will formulate the problem and its analytical solution for a multistage bunch compression system. The explicit form of the solution is given in the Appendix for the two- and the three-stage bunch compression scheme used in the existing FEL projects. At the end we will give an analytical estimation of rf tolerances and give a formula for the best compression scenario for the two-stage bunch compression system relative to these tolerances.

\section{A. Problem formulation}

In order to describe a longitudinal dynamics inside of the bunch during the compression and acceleration, let us introduce several coordinate systems.

As a starting point, we consider a bunch of electrons after the rf gun. A typical current profile and a longitudinal phase space of such a bunch are shown in Fig. 1. More details will be given later in Sec. IV. The bunch has a mean energy of several megaelectronvolts and can be already considered as a relativistic one. The relative coordinate along the bunch will be noted as $s$. It has an origin at the position of the reference particle and increases in the direction of the bunch motion: the head of the bunch has a positive value of $s$, the tail has a negative one, and the reference particle is somewhere in the middle of the bunch (a mean position of all electrons). After the rf gun the reference particle has a mean energy of the bunch $E_{0}^{0} \equiv$ $E_{0}(0)$. Here and in the following the low index " $i$ " presents a reference point along the multistage bunch compression system (after the rf gun $i \equiv 0$ ), and the upper index " 0 " shows that the quantity is taken at the position of the reference particle. Let us introduce the relative energy deviation coordinate $\delta_{0} \equiv\left(E_{0}-E_{0}^{0}\right) / E_{0}^{0}$.

In the longitudinal phase space $\left(s, \delta_{0}\right)$, the energy distribution from the gun (shown in the right plot in Fig. 1) can be approximated by a third-order polynomial,

$$
\delta_{0}(s) \equiv \delta_{0}^{\prime}(0) s+\frac{\delta_{0}^{\prime \prime}(0)}{2} s^{2}+\frac{\delta_{0}^{\prime \prime \prime}(0)}{6} s^{3},
$$

where we use a suggestion that the reference particle has a design energy: $\delta_{0}(0)=0$. Here and in the following the primes denote a partial derivative with respect to the particle position $s$ (inside the bunch after the rf gun).

Let us consider the transformation of the longitudinal phase space distribution in a multistage bunch compression and accelerating system shown in Fig. 2. The system has $N$ bunch compressors $\left(\mathrm{BC}_{1}, \ldots, \mathrm{BC}_{N}\right)$ and $N$ accelerating modules $\left(\mathrm{M}_{1}, \ldots, \mathrm{M}_{N}\right)$. The first module consists of the
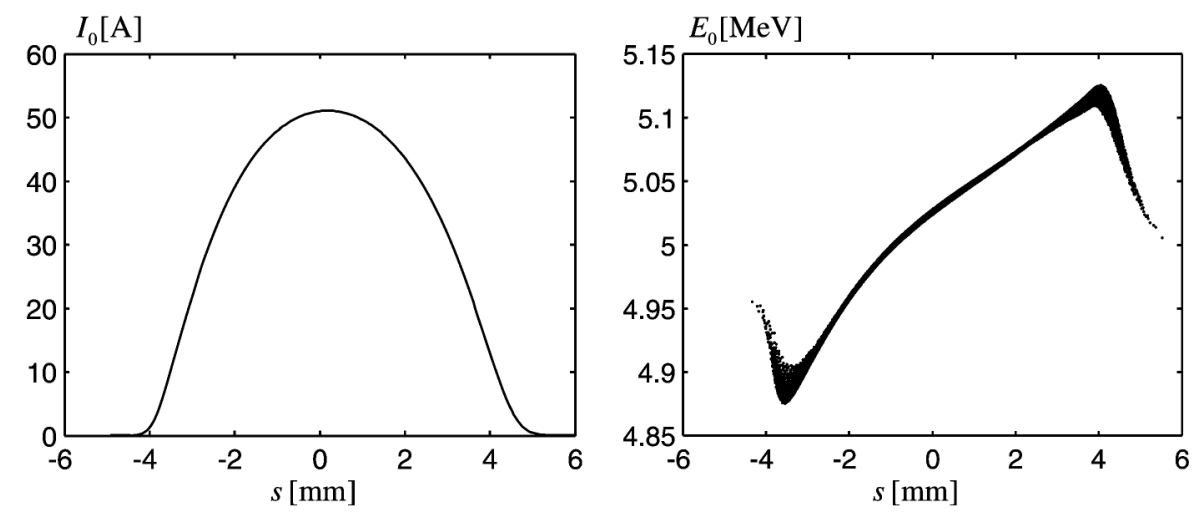

FIG. 1. The initial particle distribution after the rf gun. The left plot shows the current profile. The right plot presents the longitudinal phase space. 
fundamental harmonic module $\mathrm{M}_{1,1}$ and of the higher harmonic module $\mathbf{M}_{1, n}$ as shown in Fig. 2.

In order to describe the longitudinal beam dynamics, we introduce several additional reference points to the already described one (after the rf gun). The longitudinal coordinate after bunch compressor number $i$ will be denoted as $s_{i}$; the energy coordinate at the position immediately after the bunch compressor will be denoted as $\delta_{i}$. The reference particle is always at the origin of the coordinate system. Note that through the paper the coordinate $s$ (position in the bunch after the rf gun) will be used as an independent coordinate. All other functions depend on it. For example, the function $s_{i}(s)$ means that the particle with the initial position $s$ (in the bunch after the rf gun) has the position $s_{i}(s)$ after bunch compressor $\mathrm{BC}_{i}$. In the following we omit the dependence on coordinate $s$ in the notation.

Phase space dilution due to rf phase effects occurs because different electrons experience different rf phases as they pass through the rf modules. Thus, they gain different amounts of energy. For relativistic electrons interacting with a sinusoidally time varying field, the energy gain of the electron is proportional to the cosine of the phase angle between its position and the position of maximum energy gain [9]. Hence, the energy changes in accelerating modules $\mathrm{M}_{i}, \mathrm{M}_{1,1}$, and $\mathrm{M}_{1, n}$ can be approximated as

$$
\begin{aligned}
\Delta E_{1,1} & =e V_{1,1} \cos \left(k s+\varphi_{1,1}\right), \\
\Delta E_{1, n} & =e V_{1, n} \cos \left(n k s+\varphi_{1, n}\right), \\
\Delta E_{i} & =e V_{i} \cos \left(k s_{i-1}+\varphi_{i}\right), \quad i>1,
\end{aligned}
$$

where $e$ is the electron charge, $\varphi_{i}$ is a phase and $V_{i}$ is an oncrest voltage, $k$ is a wave number, and $n$ is a harmonic number.

The relative energy deviations in the reference points after the bunch compressors read

$$
\begin{aligned}
\delta_{1} & =\frac{(1+\delta) E_{0}^{0}+\Delta E_{1,1}+\Delta E_{1, n}}{E_{1}^{0}}-1, \\
\delta_{i} & =\frac{\left(1+\delta_{i-1}\right) E_{i-1}^{0}+\Delta E_{i}}{E_{i}^{0}}-1, \quad i=2, \ldots, N .
\end{aligned}
$$

The transformation of the longitudinal coordinate in compressor $\mathrm{BC}_{i}$ can be approximated by the expression [14]

$$
s_{i}=s_{i-1}-\left(r_{56 i} \delta_{i}+t_{56 i} \delta_{i}^{2}+u_{56 i} \delta_{i}^{3}\right), \quad i=1, \ldots, N,
$$

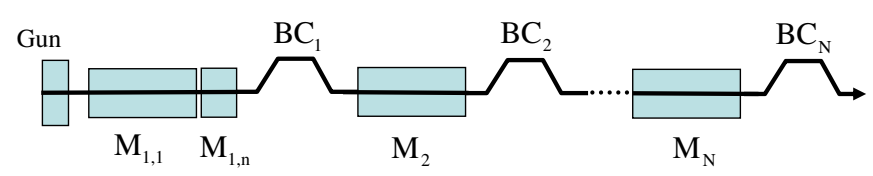

FIG. 2. The multistage bunch compression system with the high harmonic module at the first stage. where we have used a simplified notation $\left(r_{56 i} \equiv R_{56}^{(i)}\right.$, $t_{56 i} \equiv T_{566}^{(i)}, u_{56 i} \equiv U_{5666}^{(i)}$, see [14]) for the momentum compaction factors in compressor number $i$.

Equations (1)-(4) present a simple nonlinear model of the multistage bunch compression system. For the fixed values of rf parameters and momentum compaction factors, we can define a global compression function:

$$
C_{N}=\frac{1}{Z_{N}}, \quad Z_{N} \equiv \frac{\partial s_{N}}{\partial s} .
$$

The global compression function $C_{N}(s)$ presents the compression which is obtained for the particles in the neighborhood of position $s$ (the position in the bunch after the $\mathrm{rf}$ gun). For example, if we would like to increase the peak current by factor 50 in the position of the reference particle, then $C_{N}(0)=50$. In other words, function $C_{N}(s)$ describes the increase of the peak current in the slice with initial position $s$. Above we have introduced a complimentary function $Z_{N}(s)$ which we will call the inverse global compression function.

The availability of the higher harmonic module gives us the missed before 2 degrees of freedom to adjust the compression curve in the middle of the bunch. For the linear compression in the middle of the bunch we would like to have the first and the second derivatives of the global compression equal to zero: $C_{N}^{\prime 0}=0$ and $C_{N}^{\prime \prime 0}=0$. Here again the primes denote a partial derivative with respect to the particle position $s$ (inside the bunch after the rf gun) and the upper index 0 shows that the quantity is taken at the position of the reference particle.

The first and the second derivatives of the direct and inverse global compression functions are related as

$$
C_{N}^{\prime 0}=-\left(C_{N}^{0}\right)^{2} Z_{N}^{\prime 0}, \quad C_{N}^{\prime \prime 0}=2\left(C_{N}^{0}\right)^{3}\left(Z_{N}^{\prime 0}\right)^{2}-\left(C_{N}^{0}\right)^{2} Z_{N}^{\prime \prime 0} .
$$

Hence, our conditions can be reformulated as conditions on the first and the second derivatives of the inverse global compression: $Z_{N}^{\prime}=0, Z_{N}^{\prime \prime}=0$. Such a compression scenario for the FLASH facility (see Sec. IV) is shown by the dashed grey curve in Fig. 3 on the left plot. Indeed, in this case the bunch is compressed approximately by the same ratio in the middle. However, a very strong compression at the bunch head $(s>0)$ is available. The right plot in Fig. 3 presents the current profile after $\mathrm{BC}_{2}$. It was obtained from a one-dimensional tracking procedure with longitudinal space charge and wakefields included (see Sec. IV for details). A sharp spike in the current at the head of the bunch can be seen. In the general case we adjust the parameters $Z_{N}^{\prime 0}$ and $Z_{N}^{\prime \prime 0}$ to symmetrize the current profile and to avoid such spikes in the current. For, example, with the choice $Z_{N}^{\prime 0}=0, Z_{N}^{\prime \prime 0}>0$, we will have a local maximum of the global compression function $C_{N}(s)$ at the middle of the bunch and a weaker compression in the head and the tail. Such a compression scenario is shown by the solid black curves in Fig. 3. The curves in the left 

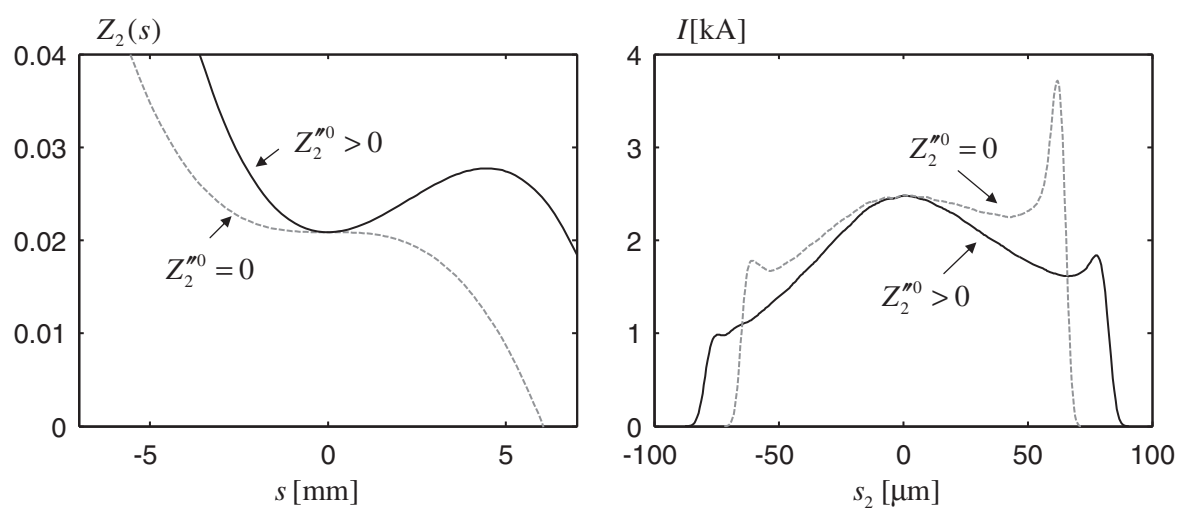

FIG. 3. The left plot presents the impact of global compression term $Z_{2}^{\prime \prime 0}$ on the compression curve along the bunch. For $Z_{2}^{\prime \prime 0}=0$ a very strong compression in the head of the bunch can be seen. The right plot shows the current profiles for these compression scenarios.

plot present the inverse function and the maximum of the compression is the local minimum in this plot.

In supplement to the global compression function, we introduce the partial compression functions $C_{i}(s)$ which describe the amount of the compression achieved after bunch compressor number $i$ :

$$
C_{i}=\frac{1}{Z_{i}}, \quad Z_{i}=\frac{\partial s_{i}}{\partial s}, \quad i=1,2, \ldots, N-1
$$

In the following we are going to use the inverse bunch compression factors as well:

$$
\begin{aligned}
Z_{i}^{0} & \equiv s_{i}^{\prime}(0), \quad Z_{i}^{\prime 0} \equiv s_{i}^{\prime \prime}(0), \\
Z_{i}^{\prime \prime 0} & \equiv s_{i}^{\prime \prime \prime}(0), \quad i=1,2, \ldots, N-1,
\end{aligned}
$$

which are defined for each reference point along the facility. Let us note that the generally used local compression factor $\bar{C}_{i}^{0}$ in BC number $i$ [14] can be written as

$$
\bar{C}_{i}^{0}=Z_{i-1}^{0} / Z_{i}^{0}
$$

The local compression factor $\bar{C}_{i}$ describes the contribution of BC number $i$ in the global compression $C_{N}^{0} \equiv C_{N}(0)$. The global compression factor $C_{N}^{0}$ and partial compression factors $C_{i}^{0} \equiv C_{i}(0)$ can be written now as a product of the local compression factors,

$$
C_{i}^{0}=\prod_{j=1}^{i} \bar{C}_{j}^{0}, \quad i=1,2, \ldots, N .
$$

Hence, the compression scenario can be described in three equivalent ways: (i) by local compression factors $\left\{\bar{C}_{i}\right\}$, (ii)

TABLE I. Parameters describing the compression schemes at DESY for nominal bunch charge of $1 \mathrm{nC}$.

\begin{tabular}{lccc}
\hline \hline Property & Symbol & Value at FLASH & Value at XFEL \\
\hline Number of compressors & $N$ & 2 & 3 \\
Initial bunch current at the reference position & $I_{0}^{0}$ & $52 \mathrm{~A}$ & $42 \mathrm{~A}$ \\
Bunch energy from the gun & $E_{0}^{0}$ & $5 \mathrm{MeV}$ & $5 \mathrm{MeV}$ \\
$\mathrm{BC}_{1}$ energy & $E_{1}^{0}$ & $130 \mathrm{MeV}$ & $130 \mathrm{MeV}$ \\
$\mathrm{BC}_{1}$ compaction factor & $r_{561}$ & $-122 \mathrm{~mm}$ & $-78 \mathrm{~mm}$ \\
$\mathrm{BC}_{1}$ compression ratio & $\bar{C}_{1}^{0}$ & 2.84 & 3 \\
Inverse compression factor after $\mathrm{BC}_{1}$ & $Z_{1}^{0}=1 / \bar{C}_{1}^{0}$ & 0.35 & 0.33 \\
$\mathrm{BC}_{2}$ energy & $E_{2}^{0}$ & $450 \mathrm{MeV}$ & $700 \mathrm{MeV}$ \\
$\mathrm{BC}_{2}$ compaction factor & $r_{562}$ & $-77.3 \mathrm{~mm}$ & $-50 \mathrm{~mm}$ \\
$\mathrm{BC}_{2}$ compression ratio & $\bar{C}_{1}^{0}$ & 16.9 & 8 \\
Inverse compression factor after $\mathrm{BC}_{2}$ & $Z_{2}^{0}=1 /\left(\bar{C}_{1}^{0} \bar{C}_{2}^{0}\right)$ & 0.02 & 0.04 \\
$\mathrm{BC}_{3}$ energy & $E_{3}^{0}$ & & $2400 \mathrm{MeV}$ \\
$\mathrm{BC}_{3}$ compaction factor & $r_{563}$ & & $-27 \mathrm{~mm}^{-1}$ \\
$\mathrm{BC}_{3}$ compression ratio & $\bar{C}_{3}^{0}$ & & 5 \\
Inverse compression factor after $\mathrm{BC}_{3}$ & $Z_{3}^{0}=1 /\left(\bar{C}_{1}^{0} \bar{C}_{2}^{0} \bar{C}_{3}^{0}\right)$ & & 0.008 \\
First derivative of the inverse compression & $Z_{N}^{\prime 0}$ & $1 \mathrm{~m}^{-1}$ & $0 \mathrm{~m}^{-1}$ \\
Second derivative of the inverse compression & $Z_{N}^{\prime \prime \prime}$ & $2000 \mathrm{~m}^{-2}$ & $1000 \mathrm{~m}^{-2}$ \\
Final bunch current at the reference position & $I_{N}^{0}=I_{0}^{0} / Z_{N}^{0}$ & $2500 \mathrm{~A}$ & $5000 \mathrm{~A}$ \\
\hline \hline
\end{tabular}




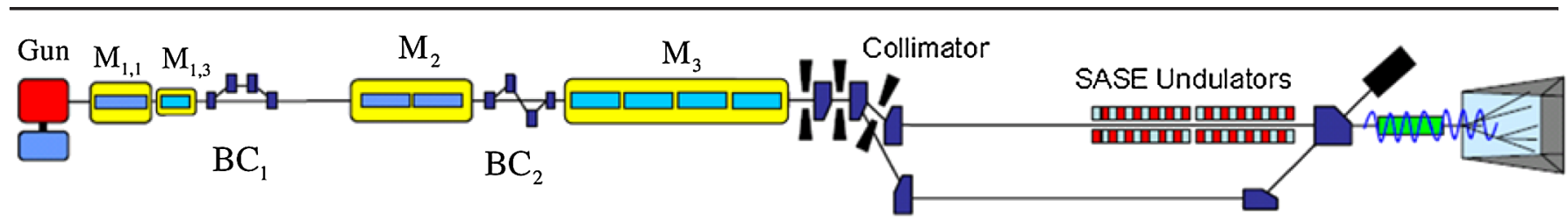

FIG. 4. The layout of the FLASH facility.

by partial compression factors $\left\{C_{i}^{0}\right\}$, and (ii) by partial inverse compression factors $\left\{Z_{i}^{0}\right\}$. Usually the first way of the description is used. However, in order to simplify the notation and to use a compact notation in the equations and in the analytical solution, we prefer to use the partial inverse compression factors $\left\{Z_{i}^{0}\right\}$ and the first and the second derivatives of the inverse global compression function: $Z_{N}^{\prime 0}$ and $Z_{N}^{\prime \prime 0}$.

In order to relate the above description to real accelerators, we list in Table I the design parameters for FLASH and European XFEL facilities and show the present layout of the FLASH facility in Fig. 4.

Let us suggest that we know the desired energies $\left\{E_{i}^{0}\right\}$ and the desired inverse compression factors $\left\{Z_{i}^{0}\right\}$ in all reference points (see Table I). Additionally we have designed the bunch compressors with the momentum compaction factors $\left\{r_{56 i}, t_{56 i}, u_{56 i}\right\}$. For symmetric $\mathrm{C}$ and $\mathrm{S}$ chicanes shown in Fig. 4, the momentum compression factors are uniquely related to the deflecting radii $\left\{r_{i}\right\}$
[14] and can be approximated analytically through the geometric parameters of the bunch compressor.

Finally, we have to decide about the desired behavior of the compression curve in the middle of the bunch and to fix the parameters of the inverse global compression $Z_{N}^{\prime 0}$ and $Z_{N}^{\prime \prime 0}$. The reasonable choice is to fix $Z_{N}^{\prime 0}$ near to zero and to choose a positive value of $Z_{N}^{\prime \prime 0}$.

At this moment we have a description of our multistage bunch compression and acceleration scheme from the viewpoint of the longitudinal beam dynamics. The next step is to convert this description in the technical values of rf parameters which have to be used in rf modules to produce the desired longitudinal dynamics.

In order to find these $2 N+2$ settings $\left(V_{1,1}, \varphi_{1,1}, V_{1, n}\right.$, $\left.\varphi_{1, n},\left\{V_{i}, \varphi_{i}\right\}, i=2,3, \ldots, N\right)$ of rf parameters in the accelerating modules, we have to solve the nonlinear system of $2 N+2$ equations,

$$
\begin{aligned}
& \delta_{1}^{0}\left(V_{1,1}, \varphi_{1,1}, V_{1,3}, \varphi_{1,3}\right)=0, \quad s_{1}^{\prime 0}\left(V_{1,1}, \varphi_{1,1}, V_{1,3}, \varphi_{1,3}\right)=Z_{1}^{0}, \\
& \delta_{i}^{0}\left(V_{1,1}, \varphi_{1,1}, V_{1,3}, \varphi_{1,3}, V_{2}, \varphi_{2}, \ldots, V_{i}, \varphi_{i}\right)=0, \quad i=2,3, \ldots, N, \\
& s_{i}^{\prime 0}\left(V_{1,1}, \varphi_{1,1}, V_{1,3}, \varphi_{1,3}, V_{2}, \varphi_{2}, \ldots, V_{i}, \varphi_{i}\right)=Z_{i}^{0}, \quad i=2,3, \ldots, N, \\
& s_{N}^{\prime \prime \prime}\left(V_{1,1}, \varphi_{1,1}, V_{1,3}, \varphi_{1,3}, V_{2}, \varphi_{2}, \ldots, V_{N}, \varphi_{N}\right)=Z_{N}^{\prime 0}, \quad s_{N}^{\prime \prime \prime}\left(V_{1,1}, \varphi_{1,1}, V_{1,3}, \varphi_{1,3}, V_{2}, \varphi_{2}, \ldots, V_{N}, \varphi_{N}\right)=Z_{N}^{\prime \prime 0},
\end{aligned}
$$

where the upper index 0 shows that the quantities are taken in the position of the reference particle $s=0$.

In the next section we describe the analytical solution of this system for an arbitrary number of stages $N$. Then in the Appendix the explicit forms of the solution for two- and three-stage bunch compression systems are given.

In the following sections we as usual omit argument $s$ and omit the upper index 0 as well. Additionally, we will use in the formulas a new symbol $\bar{E}$ for the electron energy divided by the electron charge:

$$
\bar{E}=E / e .
$$

\section{B. Analytical solution of the multistage bunch compression problem}

A straightforward solution of system (6) is possible but it is too lengthy even for two bunch compressors. We were not able to write it compactly for any number of stages. To overcome the problems we have divided the system (6) in two problems. The first system describes the longitudinal dynamics up to the reference point "1" (the position immediately after $\mathrm{BC}_{1}$ ). This is the problem for the onestage bunch compression system which was partially considered in $[11,14]$. Let us formulate and solve it.

For the time being, we suggest that we know the behavior of the energy curve in the reference point after $\mathrm{BC}_{1}$. This behavior up to the third order is fixed with the vector $\boldsymbol{\alpha}=\left(\alpha_{1}, \alpha_{2}, \alpha_{3}\right)^{T}$, where $\alpha_{i}=\frac{\partial^{i} \delta_{1}}{\partial s^{i}}$ is the $i$ th order partial derivative at the position of the reference particle.

In order to simplify the equations, let us introduce the new variables for rf parameters through the complex number notation:

$$
\begin{gathered}
X_{1, n}+i Y_{1, n}=V_{1, n} e^{i \varphi_{1, n}}, \quad X_{1,1}+i Y_{1,1}=V_{1,1} e^{i \varphi_{1,1}}, \\
X_{i}+i Y_{i}=V_{i} e^{i \varphi_{i}}, \quad i>1 .
\end{gathered}
$$

Again it is only a mathematical trick for compact notation and the reader always can convert rf parameters $\left\{X_{i}, Y_{i}\right\}$ to the commonly used rf voltages and phases $\left\{V_{i}, \varphi_{i}\right\}$ : 


$$
\begin{aligned}
V_{i} & =\left|X_{i}+i Y_{i}\right|, \quad \varphi_{i}=\arg \left(X_{i}+i Y\right), \\
X_{i} & =V_{i} \cos \varphi_{i}, \quad Y_{i}=V_{i} \sin \varphi_{i} .
\end{aligned}
$$

The first problem is a problem for four unknown rf parameters (the rf parameters before $\mathrm{BC}_{1}$ ):

$$
\begin{aligned}
& \delta_{1}\left(X_{1,1}, Y_{1,1}, X_{1, n}, Y_{1, n}\right)=0, \\
& \delta_{1}^{\prime}\left(X_{1,1}, Y_{1,1}, X_{1, n}, Y_{1, n}\right)=\alpha_{1}, \\
& \delta_{1}^{\prime \prime}\left(X_{1,1}, Y_{1,1}, X_{1, n}, Y_{1, n}\right)=\alpha_{2}, \\
& \delta_{1}^{\prime \prime \prime}\left(X_{1,1}, Y_{1,1}, X_{1, n}, Y_{1, n}\right)=\alpha_{3} .
\end{aligned}
$$

Equation (7) can be rewritten as a linear system:

$$
\begin{aligned}
& \left(\begin{array}{cccc}
1 & 0 & 1 & 0 \\
0 & -k & 0 & -n k \\
-k^{2} & 0 & -(n k)^{2} & 0 \\
0 & k^{3} & 0 & (n k)^{3}
\end{array}\right)\left(\begin{array}{c}
X_{1,1} \\
Y_{1,1} \\
X_{1, n} \\
Y_{1, n}
\end{array}\right) \\
& =\frac{1}{e}\left(\begin{array}{c}
E_{1}-E_{0} \\
E_{1} \alpha_{1}-E_{0} \delta_{0}^{\prime} \\
E_{1} \alpha_{2}-E_{0} \delta_{0}^{\prime \prime} \\
E_{1} \alpha_{3}-E_{0} \delta_{0}^{\prime \prime \prime}
\end{array}\right)
\end{aligned}
$$

If the initial values $E_{0}, \delta_{0}^{\prime}, \delta_{0}^{\prime \prime}, \delta_{0}^{\prime \prime \prime}$ and the variables $\alpha_{i}$, $i=1,2,3$, are known then the solution of Eq. (8) reads

$$
\begin{array}{ll}
X_{1,1}=\frac{F_{3}+F_{1}(k n)^{2}}{k^{2}\left(n^{2}-1\right)}, & Y_{1,1}=-\frac{F_{4}+F_{2}(k n)^{2}}{k^{3}\left(n^{2}-1\right)}, \\
X_{1, n}=-\frac{F_{3}+F_{1} k^{2}}{k^{2}\left(n^{2}-1\right)}, & Y_{1, n}=\frac{F_{4}+F_{2} k^{2}}{k^{3} n\left(n^{2}-1\right)},
\end{array}
$$

where

$$
\begin{gathered}
F_{1}=\bar{E}_{1}-\bar{E}_{0}, \quad F_{i}=\bar{E}_{1} \alpha_{i-1}-\bar{E}_{0} \frac{\partial^{i-1} \delta_{0}}{\partial s^{i-1}}, \\
i=2,3,4 .
\end{gathered}
$$

Let us formulate the second problem which describes the longitudinal dynamics from the reference point " 1 " (the position immediately after $\mathrm{BC}_{1}$ ) to the end of the compression scheme.

The second problem is a problem for $2 N+1$ variables: $2 N-2$ rf parameters for accelerator modules between compressors and three components of vector $\boldsymbol{\alpha}=$ $\left(\alpha_{1}, \alpha_{2}, \alpha_{3}\right)^{T}$ :

$$
\begin{aligned}
& \delta_{i}(\mathbf{X})=0, \quad i=2,3, \ldots, N, \\
& s_{i}^{\prime}\left(\mathbf{X}, \mathbf{Y}, \alpha_{1}\right)=Z_{i}, \quad i=1,2, \ldots, N, \\
& s_{N}^{\prime \prime}\left(\mathbf{X}, \mathbf{Y}, \alpha_{1}, \alpha_{2}\right)=Z_{N}^{\prime}, \quad s_{N}^{\prime \prime \prime}(\mathbf{X}, \mathbf{Y}, \boldsymbol{\alpha})=Z_{N}^{\prime \prime},
\end{aligned}
$$

where $\mathbf{X}=\left(X_{2}, \ldots, X_{N}\right)^{T}, \mathbf{Y}=\left(Y_{2}, \ldots, Y_{N}\right)^{T}$ are vectors of the rf parameters and set $\left\{\alpha_{i}\right\}$ describes the energy curve immediately after the high harmonic module as was already mentioned above.

Let us describe the solution of system (10) step by step. At the beginning, from the first $N-1$ equations, $\delta_{i}(\mathbf{X})=0$, we can easily find the components of vector $\mathbf{X}$ :

$$
X_{i}=\bar{E}_{i}-\bar{E}_{i-1}, \quad i=2, \ldots, N .
$$

From the next $N$ equations, $s_{i}^{\prime}\left(\mathbf{X}, \mathbf{Y}, \alpha_{1}\right)=Z_{i}, i=$ $1, \ldots, N$, we find the components of vector $\mathbf{Y}$ and the energy chirp $\alpha_{1} \equiv \delta_{1}^{\prime}$ before $\mathrm{BC}_{1}$ :

$$
\begin{gathered}
\delta_{i}^{\prime}=\frac{Z_{i-1}-Z_{i}}{r_{56 i}}, \quad i=1, \ldots, N, \\
Y_{i}=\frac{\delta_{i-1}^{\prime} \bar{E}_{i-1}-\delta_{i}^{\prime} \bar{E}_{i}}{k Z_{i-1}}, \quad i=2, \ldots, N .
\end{gathered}
$$

At this point we have defined rf parameters $\left\{X_{i}, Y_{i}\right\}, i=$ $2,3, \ldots, N$, for all stages except the first one.

In order to write explicitly the last two equations in system (10), we need to find the first three derivatives of functions $s_{i}(s)$ and $\delta_{i}(s)$. In the following we as usual omit argument $s$. In this simplified notation the first three derivatives at $s=0$ read

$$
\begin{aligned}
s_{i}^{\prime} & =s_{i-1}^{\prime}-r_{56 i} \delta_{i}^{\prime}, \quad s_{i}^{\prime \prime}=s_{i-1}^{\prime \prime}-r_{56 i} \delta_{i}^{\prime \prime}-2 t_{56 i}\left(\delta_{i}^{\prime}\right)^{2}, \quad s_{i}^{\prime \prime \prime}=s_{i-1}^{\prime \prime \prime}-r_{56 i} \delta_{i}^{\prime \prime \prime}-6 t_{56 i} \delta_{i}^{\prime} \delta_{i}^{\prime \prime}-6 u_{56 i}\left(\delta_{i}^{\prime}\right)^{3}, \quad i=1,2, \ldots, N, \\
s_{0}^{\prime} & \equiv 1, \quad s_{0}^{\prime \prime} \equiv 0, \quad s_{0}^{\prime \prime \prime} \equiv 0, \quad \delta_{i}^{\prime}=\frac{\delta_{i-1}^{\prime} \bar{E}_{i-1}-k Z_{i-1} Y_{i}}{\bar{E}_{i}}, \quad \delta_{i}^{\prime \prime}=\frac{\delta_{i-1}^{\prime \prime} \bar{E}_{i-1}-k^{2} Z_{i-1}^{2} X_{i}-k Z_{i-1}^{\prime} Y_{i}}{\bar{E}_{i}} \\
\delta_{i}^{\prime \prime \prime} & =\frac{\delta_{i-1}^{\prime \prime \prime} \bar{E}_{i-1}-k^{3} Z_{i-1}^{3} Y_{i}-3 k^{2} Z_{i-1} Z_{i-1}^{\prime} X_{i}-k Z_{i-1}^{\prime \prime} Y_{i}}{\bar{E}_{i}}, \quad i=2,3, \ldots, N
\end{aligned}
$$

Finally, we describe the most involved part of the solution. From equation $s_{N}^{\prime \prime}\left(\mathbf{X}, \mathbf{Y}, \alpha_{1}, \alpha_{2}\right)=Z_{N}^{\prime}$ we can find parameter $\alpha_{2}$. This equation can be rewritten as a system of linear difference equations [see Eqs. (10) and (14)]

$$
\begin{aligned}
& x_{i}=x_{i-1}+a_{i} y_{i}+b_{i}, \quad i=1, \ldots, N, \\
& y_{i}=y_{i-1}+d_{i} x_{i-1}+e_{i}, \quad i=2, \ldots, N, \\
& x_{0}=0, \quad x_{N}=x_{N}^{0},
\end{aligned}
$$

where

$$
\begin{aligned}
& x_{i} \equiv s_{i}^{\prime \prime}, \quad y_{i} \equiv \bar{E}_{i} \delta_{i}^{\prime \prime}, \quad x_{N}^{0} \equiv Z_{N}^{\prime}, \quad a_{i}=-\frac{r_{56 i}}{E_{i}}, \\
& b_{i}=-2 t_{56 i}\left(\delta_{i}^{\prime}\right)^{2}, \quad i=1, \ldots, N, \quad d_{i}=-k Y_{i}, \\
& e_{i}=-k^{2} Z_{i-1}^{2} X_{i}, \quad i=2, \ldots, N .
\end{aligned}
$$

It is easy to check that the solution of the problem (15) can be found as

$$
\alpha_{2}=\frac{y_{1}}{\bar{E}_{1}}, \quad y_{1}=\frac{Z_{N}^{\prime}-\tilde{x}_{N}}{\bar{x}_{N}},
$$


where $\bar{x}_{N}$ and $\tilde{x}_{N}$ are solutions of the particular homogeneous and inhomogeneous problems,

$$
\begin{aligned}
& \bar{x}_{i}=\bar{x}_{i-1}+a_{i} \bar{y}_{i}, \quad \bar{y}_{i}=\bar{y}_{i-1}+d_{i} \bar{x}_{i-1}, \quad \bar{x}_{0}=0, \quad \bar{y}_{1}=1, \quad i=1, \ldots, N, \\
& \tilde{x}_{i}=\tilde{x}_{i-1}+a_{i} \tilde{y}_{i}+b_{i}, \quad \tilde{y}_{i}=\tilde{y}_{i-1}+d_{i} \tilde{x}_{i-1}+e_{i}, \quad \tilde{x}_{0}=0, \quad \tilde{y}_{1}=0, \quad i=1, \ldots, N \text {. }
\end{aligned}
$$

The unknowns $\tilde{x}_{N}$ and $\bar{x}_{N}$ can be found straightforwardly from the recurrence relations (18).

Finally, the last equation, $s_{N}^{\prime \prime \prime}(\mathbf{X}, \mathbf{Y}, \boldsymbol{\alpha})=Z_{N}^{\prime \prime}$, allows one to find $\alpha_{3}$. This equation can be rewritten in a system of linear difference equations like (15) with some of the coefficients being different:

$$
\begin{aligned}
& x_{i} \equiv s_{i}^{\prime \prime \prime}, \quad y_{i} \equiv \bar{E}_{i} \delta_{i}^{\prime \prime \prime}, \quad x_{N}^{0} \equiv Z_{N}^{\prime \prime}, \\
& b_{i}=-6 t_{56 i} \delta^{\prime} \delta^{\prime \prime}-6 u_{56 i}\left(\delta_{i}^{\prime}\right)^{3}, \\
& e_{i}=k^{3} Z_{i-1}^{3} Y_{i}-3 k^{2} Z_{i-1}^{2} Z_{i-1}^{\prime} X_{i} .
\end{aligned}
$$

Unknown $\alpha_{3}$ can be written as

$$
\alpha_{3}=\frac{y_{1}}{\bar{E}_{1}}, \quad y_{1}=\frac{Z_{N}^{\prime \prime}-\tilde{x}_{N}}{\bar{x}_{N}},
$$

where $\bar{x}_{N}$ and $\tilde{x}_{N}$ are solutions of the homogeneous and the inhomogeneous problems of the form (18) but with coefficients given by Eq. (19).

Above we have found a unique solution of the original problem (6) for any number of stages $N$. The reader can argue that the solution looks complicated and perhaps it will be better to consider only two- and three-stage compression schemes. However, as it can be seen from the Appendix that even for two stages of compression the explicit form of the solution does not look simpler. We will use this analytical solution in Sec. IV to define a bunch compression working point for the FLASH facility [1] and it is used in [15] to define the working points for the European XFEL facility [4].

\section{Analytical estimation of rf tolerances}

The final bunch length and the peak current are sensitive to the energy chirp and thus to the precise values of the $\mathrm{rf}$ parameters. Let us calculate a change of the compression due to a change of the rf parameters. Unlike other publications on rf tolerances (for example, $[11,14,16]$ ), we do not consider the voltage and the phase tolerances separately. Such one-dimensional treatment is replaced in this work by two-dimensional consideration where the phase and the voltage can change simultaneously and we estimate the tolerance from the worst possible direction of the change (the maximal sensitivity to the simultaneous deviation of the rf parameters, see Fig. 5). In such treatment no compensation in the rf tolerance can be found (see Fig. 8 and $[11,16])$.

Before starting with mathematical formalism let us describe the main idea on the example of rf tolerance in module $\mathrm{M}_{1,1}$. The left plot in Fig. 5 presents the chosen rf parameters,

$$
X_{1,1}^{0}=V_{1,1}^{0} \cos \varphi_{1,1}^{0}, \quad Y_{1,1}^{0}=V_{1,1}^{0} \sin \varphi_{1,1}^{0},
$$

as a point in the parameter space $\left(X_{11}, Y_{11}\right)$. Here and in the following, symbol 0 stays for the rf parameters as obtained in Sec. II B from the analytical solution. Vectors $\mathbf{v}_{\Delta V}$ and $\mathbf{v}_{\Delta \varphi}$ define the directions of the rf parameters shift, when we change only the voltage $V_{1,1}$ or only the phase $\varphi_{1,1}$, correspondingly. The contour plot in Fig. 5 presents change of the relative compression $\left|\Delta C_{2}\right| / C_{2}$ near the working point (as obtained for FLASH with parameters of Table I). Here we see that the most critical direction of the steepest
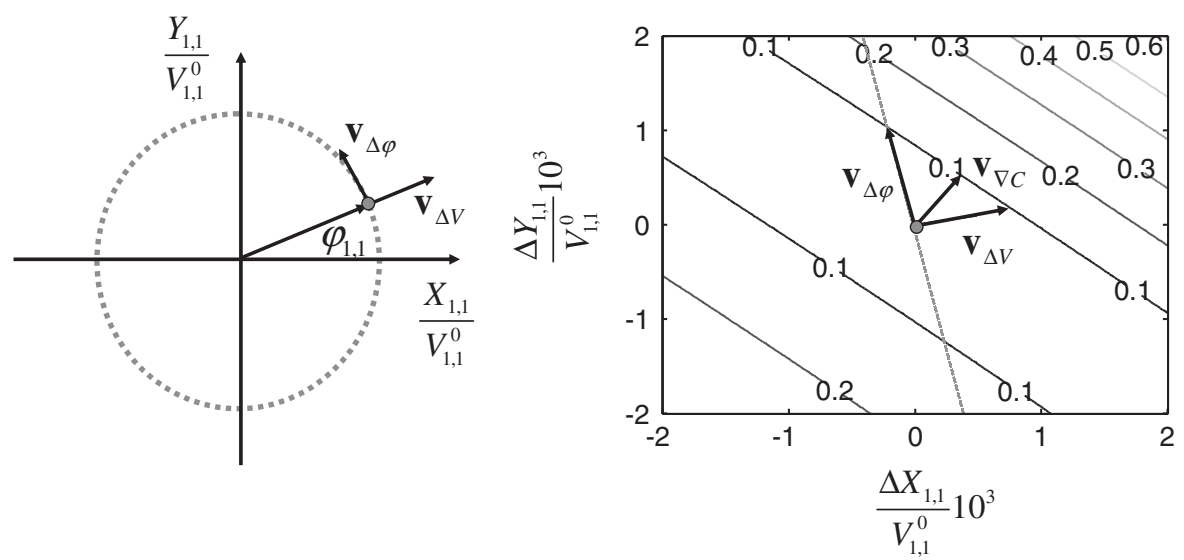

FIG. 5. The left plot presents the rf parameters of module $\mathrm{M}_{1,1}$ as a point in the parameter space $\left(X_{11}, Y_{11}\right)$. The contour plot at the right-hand side presents the change of the relative compression $\left|\Delta C_{2}\right| / C_{2}$ near the working point at FLASH. 
change of the compression is given by gradient vector $\mathbf{v}_{\nabla C}$. In the following we derive analytical expressions for estimation of the gradient vector $\nabla C_{N}$ in two- and three-stage compression schemes.

To simplify the notation we define

$$
X_{1}=X_{1,1}+X_{1, n}, \quad Y_{1}=Y_{1,1}+n Y_{1, n} .
$$

Additionally we introduce rf parameter vectors

$$
\begin{array}{rlrl}
\mathbf{v}_{i} & \equiv\left(X_{i}, Y_{i}\right)^{T}, & \mathbf{v}_{i}^{0} \equiv\left(X_{i}^{0}, Y_{i}^{0}\right)^{T}, \quad i=2,3, \ldots, N, \\
\mathbf{v}_{1,1} \equiv\left(X_{1,1}, Y_{1,1}\right)^{T}, & \mathbf{v}_{1,1}^{0} \equiv\left(X_{1,1}^{0}, Y_{1,1}^{0}\right)^{T}, \\
\mathbf{v}_{1, n} \equiv\left(X_{1, n}, Y_{1, n}\right)^{T}, & & \mathbf{v}_{i}^{0} \equiv\left(X_{i}^{0}, Y_{i}^{0}\right)^{T},
\end{array}
$$

where the distortion vectors are defined as

$$
\begin{aligned}
\Delta \mathbf{v}_{i} & \equiv\left(\Delta X_{i}, \Delta Y_{i}\right)^{T}, \quad i=1,2, \ldots, N, \\
\Delta \mathbf{v}_{1,1} & \equiv\left(\Delta X_{1,1}, \Delta Y_{1,1}\right)^{T}, \quad \Delta \mathbf{v}_{1, n} \equiv\left(\Delta X_{1, n}, \Delta Y_{1, n}\right)^{T}, \\
\Delta X_{1} & =\Delta X_{1,1}+\Delta X_{1, n} .
\end{aligned}
$$

For example, the distortion vector $\Delta \mathbf{v}_{1,1}$ for the module $M_{1,1}$ has components

$$
\begin{aligned}
\Delta X_{1,1} & =X_{1,1}-X_{1,1}^{0} \\
& =\left(V_{1,1}^{0}+\Delta V_{1,1}\right) \cos \left(\varphi_{1,1}^{0}+\Delta \varphi_{1,1}\right)-V_{1,1}^{0} \cos \left(\varphi_{1,1}^{0}\right), \\
\Delta Y_{1,1} & =Y_{1,1}-Y_{1,1}^{0} \\
& =\left(V_{1,1}^{0}+\Delta V_{1,1}\right) \sin \left(\varphi_{1,1}^{0}+\Delta \varphi_{1,1}\right)-V_{1,1}^{0} \sin \left(\varphi_{1,1}^{0}\right) .
\end{aligned}
$$

In order to obtain a stable bunch compression, we require that the relative change of the global compression $C_{N} \equiv Z_{N}^{-1}$ at $s=0$ is smaller than $\Theta$ :

$$
\left|\frac{C_{N}(\mathbf{v})-C_{N}\left(\mathbf{v}^{0}\right)}{C_{N}\left(\mathbf{v}^{0}\right)}\right| \leq \Theta .
$$

Neglecting the second-order terms the last inequality can be rewritten in the form

$$
\left|\Delta \mathbf{v} \cdot \nabla_{\mathbf{v}} C_{N}(\mathbf{v})\right| \leq C_{N}\left(\mathbf{v}^{0}\right) \Theta,
$$

where term $\nabla_{\mathbf{v}} C_{N}=\left(\partial_{X} C_{N}, \partial_{Y} C_{N}\right)^{T}$ means the gradient of the compression in two-dimensional space of rf parameters $(X \equiv V \cos \varphi, Y \equiv V \sin \varphi) . \quad$ Applying the CauchyBunyakovsky inequality, we obtain that the admissible relative distortion $|\Delta \mathbf{v}|\left|\mathbf{v}^{0}\right|^{-1}$ in $\operatorname{rf}$ parameters $(X, Y)$ has to fulfill the inequality

$$
\frac{|\Delta \mathbf{v}|}{\left|\mathbf{v}^{0}\right|} \leq \frac{\Theta}{V C_{N}\left|\nabla_{\mathbf{v}} Z_{N}\right|},
$$

where we have used the relations

$$
C_{N}^{-1} \nabla_{\mathbf{v}} C_{N}=-Z_{N}^{-1} \nabla_{\mathbf{v}} Z_{N}, \quad Z_{N} \equiv C_{N}^{-1} .
$$

Following relation (21) let us define the rf tolerance as

$$
\theta_{\mathbf{v}}=\frac{\Theta}{V C_{N}\left|\nabla_{\mathbf{v}} Z_{N}\right|}
$$

In the same manner we can define voltage and phase tolerances by relations

$$
\theta_{V}=\frac{\Theta}{V C_{N}\left|\partial_{V} Z_{N}\right|}, \quad \theta_{\varphi}=\frac{\Theta}{C_{N}\left|\partial_{\varphi} Z_{N}\right|} .
$$

The rf tolerance $\theta_{\mathbf{v}}$ introduced above is related to the voltage and the phase tolerance by the simple expression

$$
\theta_{\mathbf{v}}=\frac{\theta_{V} \theta_{\varphi}}{\sqrt{\theta_{V}^{2}+\theta_{\varphi}^{2}}} .
$$

Let us discuss the meaning of Eqs. (22)-(24) and their relations. Equation (22) means that the change of the relative compression will be less than $\Theta$ if the relative length change of the rf vector $\mathbf{v}=(X, Y)^{T}$ is less than $\theta_{\mathbf{v}}$. Equation (23) means that the change of the relative compression will be less than $\Theta$ if the relative change of the voltage $V$ alone is less than $\theta_{V}$ or the change of the phase $\varphi$ alone is less than $\theta_{\varphi}$. Finally, if we have estimated the admissible change $\theta_{V}$ of the voltage alone and have estimated the admissible change of the phase $\theta_{\varphi}$ alone, then the admissible change of both parameters simultaneously is given by Eq. (24).

In order to estimate the rf tolerances, we need to estimate the partial derivatives relative to the rf parameters. Let us denote by a point over the symbol the partial derivative with respect to a rf parameter. Then the partial derivative of inverse compression $Z_{i}$ after stage $i$ can be found by relations

$$
\begin{aligned}
\dot{Z}_{i} & =\dot{Z}_{i-1}-r_{56 i} \dot{\delta}_{i}^{\prime}-2 t_{56 i} \delta_{i}^{\prime} \dot{\delta}_{i}, \\
\bar{E}_{i} \dot{\delta}_{i}^{\prime} & =\bar{E}_{i-1} \dot{\delta}_{i-1}^{\prime}-k Z_{i-1} \dot{Y}_{i}-k^{2} X_{i} Z_{i-1} \dot{s}_{i-1}-k Y_{i} \dot{Z}_{i-1}, \\
\bar{E}_{i} \dot{\delta}_{i} & =\bar{E}_{i-1} \dot{\delta}_{i-1}+\dot{X}_{i}-k Y_{i} \dot{s}_{i-1}, \dot{s}_{i}=\dot{s}_{i-1}-r_{56 i} \dot{\delta}_{i} .
\end{aligned}
$$

The partial derivatives of the compression with respect to $\mathrm{rf}$ parameters $\left(X_{1}, Y_{1}\right)$ immediately after compressor $\mathrm{BC}_{2}$ read

$$
\begin{aligned}
\partial_{X_{1}} Z_{2} & =\partial_{X_{1}} Z_{1}-r_{562} \partial_{X_{1}} \delta_{2}^{\prime}-2 t_{562} \delta_{2}^{\prime} \partial_{X_{1}} \delta_{2}, \\
\delta_{2}^{\prime} & =\frac{Z_{1}-Z_{2}}{r_{562}}, \quad \bar{E}_{2} \partial_{X_{1}} \delta_{2}=1+k Y_{2} \frac{r_{561}}{\bar{E}_{1}}, \\
\bar{E}_{2} \partial_{X_{1}} \delta_{2}^{\prime} & =k^{2} X_{2} Z_{1} \frac{r_{561}}{\bar{E}_{1}}-k Y_{2} \partial_{X_{1}} Z_{1}, \\
\partial_{Y_{1}} Z_{2} & =\partial_{Y_{1}} Z_{1}-r_{562} \partial_{Y_{1}} \delta_{2}^{\prime}, \quad \bar{E}_{2} \partial_{Y_{1}} \delta_{2}^{\prime}=-k-k Y_{2} \partial_{Y_{1}} Z_{1} .
\end{aligned}
$$

The partial derivatives of the compression with respect to of parameters $\left(X_{1}, Y_{1}\right)$ immediately after compressor $\mathrm{BC}_{3}$ can be found from relations 


$$
\begin{aligned}
\partial_{X_{1}} Z_{3} & =\partial_{X_{1}} Z_{2}-r_{563} \partial_{X_{1}} \delta_{3}^{\prime}-2 t_{563} \delta_{3}^{\prime} \partial_{X_{1}} \delta_{3}, \quad \delta_{3}^{\prime}=\frac{Z_{2}-Z_{3}}{r_{563}}, \quad \bar{E}_{3} \partial_{X_{1}} \delta_{3}=\bar{E}_{2} \partial_{X_{1}} \delta_{2}-k Y_{3} \partial_{X_{1}} s_{2}, \\
\partial_{X_{1}} s_{2} & =-\frac{r_{561}}{\bar{E}_{1}}-r_{562} \partial_{X_{1}} \delta_{2}, \quad \bar{E}_{3} \partial_{X_{1}} \delta_{3}^{\prime}=\bar{E}_{2} \partial_{X_{1}} \delta_{2}^{\prime}-k^{2} X_{3} Z_{2} \partial_{X_{1}} s_{2}-k Y_{3} \partial_{X_{1}} Z_{2}, \quad \partial_{Y_{1}} Z_{3}=\partial_{Y_{1}} Z_{2}-r_{563} \partial_{Y_{1}} \delta_{3}^{\prime}, \\
\bar{E}_{3} \partial_{Y_{1}} \delta_{3}^{\prime} & =\bar{E}_{2} \partial_{Y_{1}} \delta_{2}^{\prime}-k Y_{3} \partial_{Y_{1}} Z_{2} .
\end{aligned}
$$

It follows from Eq. (20) that the partial derivatives with respect to the rf parameters in modules $\mathrm{M}_{1,1}$ and third harmonic module $\mathrm{M}_{1,3}$ are given by relations

$$
\begin{array}{ll}
\partial_{X_{1,1}} Z_{i}=\partial_{X_{1}} Z_{i}, & \partial_{Y_{1,1}} Z_{i}=\partial_{Y_{1}} Z_{i}, \\
\partial_{X_{1, n}} Z_{i}=\partial_{X_{1}} Z_{i}, & \partial_{Y_{1, n}} Z_{i}=n \partial_{Y_{1}} Z_{i} .
\end{array}
$$

In the following we are going to consider rf tolerances only in the first fundamental module $\mathrm{M}_{1,1}$. Usually this tolerance is the strongest one. The expressions for other tolerances can be found in [17].
In order to estimate the partial derivatives of the compression with respect to the voltages or the phases, we can use the relations

$$
\begin{aligned}
& \partial_{V_{j}} Z_{i}=\partial_{X_{j}} Z_{i} \cos \varphi_{j}+\partial_{Y_{j}} Z_{i} \sin \varphi_{j}, \\
& \partial_{\varphi_{j}} Z_{i}=V_{j}\left(-\partial_{X_{j}} Z_{i} \sin \varphi_{j}+\partial_{Y_{j}} Z_{i} \cos \varphi_{j}\right) .
\end{aligned}
$$

The admissible distortion in rf parameters in module $\mathrm{M}_{1,1}$ for two-stage bunch compression scheme can be estimated as

$$
\begin{aligned}
\theta_{\mathbf{v}_{1,1}} & =\frac{\Theta}{V_{1,1}^{0} C_{2}\left|\nabla_{\mathbf{v}_{1,1}} Z_{2}\right|}, \quad\left|\nabla_{\mathbf{v}_{1,1}} Z_{2}\right|=k \frac{\left|r_{561} r_{562}\right|}{\bar{E}_{1} \bar{E}_{2}} \sqrt{A_{2}^{2}+B_{2}^{2}}, \quad A_{2}=\left(\frac{\bar{E}_{2}}{r_{562}}+\frac{\bar{E}_{1}}{r_{561}}+k Y_{2}\right), \\
B_{2} & =\left(k X_{2} Z_{1}+2 \frac{t_{561}}{r_{561}}\left(\frac{\bar{E}_{2}}{r_{562}}+k Y_{2}\right) \frac{\delta_{1}^{\prime}}{k}+2 \frac{t_{562}}{r_{562}}\left(\frac{\bar{E}_{1}}{r_{561}}+k Y_{2}\right) \frac{\delta_{2}^{\prime}}{k}\right) .
\end{aligned}
$$

If we neglect the nonlinear compression terms and use Eq. (27), then we can write the simple estimations for maximal admissible distortion in $\mathrm{rf}$ vector in $\mathrm{M}_{1,1}$ :

$$
\theta_{\mathbf{v}_{1,1}}=\frac{\Theta \bar{E}_{1} \bar{E}_{2}}{k V_{1,1}^{0} C_{2} C_{1} \sqrt{\left(\bar{E}_{1} r_{562}+\bar{E}_{2} r_{561} C_{2}^{-1}\right)^{2}+r_{561}^{2} r_{562}^{2} k^{2}\left[\bar{E}_{2}-\bar{E}_{1}\right]^{2} C_{1}^{-4}}} .
$$

For the three-stage bunch compression scheme, the estimation for maximal admissible distortion in $\mathrm{rf}$ vector in module $\mathrm{M}_{1,1}$ reads (we neglect again nonlinear compression terms)

$$
\begin{aligned}
\theta_{\mathbf{v}_{1,1}}= & \frac{\Theta \bar{E}_{1} \bar{E}_{2} \bar{E}_{3}}{k V_{1,1}^{0} C_{3} C_{2} C_{1} \sqrt{A_{3}^{2}+B_{3}^{2}}}, \\
A_{3}= & r_{561} \bar{E}_{2} \bar{E}_{3} C_{2}^{-1} C_{3}^{-1}+r_{562} \bar{E}_{1} \bar{E}_{3} C_{3}^{-1}+r_{563} \bar{E}_{1} \bar{E}_{2} C_{1}^{-1}, \\
B_{3} \approx & k\left[r_{561}\left(\bar{E}_{2}-\bar{E}_{1}\right) C_{1}^{-2}\left(\bar{E}_{3} r_{562} C_{3}^{-1}+\bar{E}_{2} r_{563} C_{1}^{-1}\right)\right. \\
& \left.+r_{563}\left(\bar{E}_{3}-\bar{E}_{2}\right) C_{2}^{-2}\left(\bar{E}_{2} r_{561} C_{2}^{-1}+\bar{E}_{1} r_{562}\right)\right] .
\end{aligned}
$$

Finally, let us consider a question about the best compression scenario from the point of view of the best possible tolerance in module $\mathrm{M}_{1,1}$. We consider the two-stage bunch compression scheme and use Eq. (28) to find the best value of $C_{1}$ for the fixed value of $C_{2}$. From the condition

$$
\frac{\partial}{\partial Z_{1}}\left|\nabla_{\mathbf{v}_{1,1}} Z_{2}\right|=0
$$

it is easy to find the optimal value of the compression in the first bunch compressor:

$$
C_{1}=\sqrt{\frac{k r_{561} r_{562}\left(\bar{E}_{2}-\bar{E}_{1}\right)}{-r_{562} \bar{E}_{1}-r_{561} \bar{E}_{2} C_{2}^{-1}}}
$$

For example, if we take the values from Table I for FLASH, then we obtain that the most stable compression scenario will be obtained by choosing compression factor $C_{1}=2.7$ (see Fig. 9).

As will be shown in the next sections, the last equation gives practical advice for choosing the local compression factor $C_{1}=Z_{1}^{-1}$ in the first bunch compressor. Choosing such compression, for example, in FLASH allows for increasing the rf tolerance by factor 3 compared to other scenarios (see Fig. 9).

In the example of Sec. IV, we consider as an optimization parameter the $\mathrm{rf}$ tolerance $\theta_{\mathbf{v}}$ alone. It defines the admissible change of the relative length of the rf vector $\mathbf{v}=(V \cos \varphi, V \sin \varphi)^{T}$. In the real rf systems it can be difficult to control the phase and the amplitude with the same accuracy. In this case we can introduce weighted tolerances for them. It is easy to see that Eq. (21) will be satisfied (for small changes of the amplitude and the phase) if 


$$
\begin{aligned}
& \frac{|\Delta V|}{V^{0}} \leq \sqrt{\beta_{1}} \theta_{\mathbf{v}}, \quad|\Delta \varphi| \leq \sqrt{\beta_{2}} \theta_{\mathbf{v}}, \quad \beta_{1}+\beta_{2}=1, \\
& 0 \leq \beta_{1} \leq 1, \quad 0 \leq \beta_{2} \leq 1 \text {, }
\end{aligned}
$$

where the weights $\left\{\beta_{i}\right\}$ are related to the difficulties to control the rf parameters.

\section{MULTISTAGE BUNCH COMPRESSION WITH COLLECTIVE EFFECTS}

\section{A. Collective effects and tracking codes}

The analytical solution introduced before neglects the collective effects in the main beam line. In order to take them into account, we do tracking simulations taking into account the collective effects through analytical estimations (space-charge forces, wakefields), or through direct numerical solution with tracking codes.

To take into account coherent synchrotron radiation (CSR) in bunch compressors, we use code CSRTRACK [18]. This code tracks particle ensembles through beam lines with arbitrary geometry. It offers different algorithms for the field calculation: from the fast "projected" 1D method [19] to the most rigorous one, the threedimensional integration over 3D Gaussian sub-bunch distributions [20].

For high peak currents the compression is affected by wakefields from the vacuum chamber and by space-charge forces. The free space longitudinal space-charge impedance and the corresponding wake function for bunch with Gaussian transverse profile are given by [21]

$$
\begin{aligned}
\frac{d Z(\omega)}{d z}= & i \frac{Z_{0}}{2 \gamma^{2}} \frac{\omega}{c}\left[\frac{1}{2 \pi} e^{\alpha^{2}} \Gamma\left(0, \alpha^{2}\right)\right], \quad \alpha=\frac{\omega \sigma_{\perp}}{c \gamma}, \\
\Gamma(0, \alpha)= & \int_{x}^{\infty} \frac{e^{-t}}{t} d t . \\
\frac{d w(s)}{d z}= & \theta(s) \frac{Z_{0} c}{8 \pi \sigma_{\perp}^{2}}\left[\frac{\xi(s)}{|\xi(s)|}\right. \\
& \left.-\frac{\sqrt{\pi}}{2} \xi(s) e^{\left(\xi(s)^{2}\right) / 4} \operatorname{Erfc}\left[\frac{|\xi(s)|}{2}\right]\right], \\
\xi(s)= & \frac{s \gamma}{\sigma_{\perp}}
\end{aligned}
$$

where $\sigma_{\perp}$ is the transverse rms size of the beam, $\theta(s)$ is the Heaviside step function, $Z_{0}$ is the free space impedance, and $c$ is the vacuum light velocity.

Let us consider the bunch accelerated from energy $\gamma_{0}$ to the energy $\gamma_{1}$ along distance $L$. We use an adiabatic approximation which takes into account a slow change of the rms size of the bunch during the acceleration:

$$
\begin{aligned}
& Z(\omega)=\int_{0}^{L} \frac{d Z\left(\omega, r_{b}, \gamma\right)}{d z} d z=i \frac{\omega Z_{0}}{4 \pi c} \int_{0}^{L} \frac{e^{\alpha(z)^{2}} \Gamma\left[0, \alpha(z)^{2}\right]}{\gamma(z)^{2}} d z, \\
& \alpha(z)=\frac{\omega \sigma_{\perp}(z)}{c \gamma(z)}, \quad \sigma_{\perp}(z)=\sqrt{\frac{\varepsilon_{n}\langle\beta\rangle}{\gamma(z)}} \\
& \gamma(z)=\gamma_{0}+\frac{\gamma_{1}-\gamma_{0}}{L} z,
\end{aligned}
$$

where $\langle\beta\rangle$ is the averaged optical beta function along distance $L, \varepsilon_{n}$ is the normalized transverse emittance.

Along with the above analytical estimations we use an alternative approach based on the straightforward tracking with code ASTRA [22]. This program tracks particles through user defined external fields taking into account the space-charge field of the particle cloud.

Both codes, CSRTRACK and ASTRA, do tracking in free space neglecting the impact of the vacuum chamber on the self-fields. We use coupling impedances (or wake functions) to take into account interactions of the bunch with the boundary. The wakefield code ECHO [23] was used to estimate the wake functions of different beam line elements.

The FLASH facility contains 56 TESLA accelerating cavities. Their wake function is given by [24]

$$
w(s)=10^{12} \theta(s) 43 e^{-24 \sqrt{s}} .
$$

The wake function of the harmonic module with four cavities reads [25]

$$
\begin{aligned}
w(s)= & 10^{12} \theta(s)\left(318 e^{-34.5 \sqrt{s}}\right. \\
& \left.+0.9 \frac{\cos \left(5830 s^{0.83}\right)}{\sqrt{s}+195 s}+0.036 \delta(s)\right),
\end{aligned}
$$

where the last term with the Dirac delta function describes the reduction of the pipe radius from 39 to $20 \mathrm{~mm}$ at the position of the third harmonic module.

\section{B. An iterative tracking procedure with collective effects}

The analytical solution for rf parameters given in Sec. II will not produce the required compression in reality. The strong self-fields can severely deteriorate the properties of the compressed bunch. In order to take the collective effects into account, we have to carry out the tracking simulations. For the adjustment of the rf parameters we use an iterative procedure, which starts from the values of the rf parameters obtained through the analytical solution introduced in Sec. II.

The rf parameters for the compression scenario without self-fields can be found from Eq. (6) which in operator form reads

$$
\mathbf{A}_{0}(\mathbf{x})=\mathbf{f}_{0}
$$


where nonlinear operator $\mathbf{A}_{0}(\cdot)$ is defined from Eq. (6) and the right-hand side $\mathbf{f}_{0}$ and the unknown vector of the $\mathrm{rf}$ parameters $\mathbf{x}$ are given by relations (for two-stage BC system)

$$
\begin{aligned}
& \mathbf{f}_{0}=\left(E_{1}, E_{2}, Z_{1}, Z_{2}, Z_{2}^{\prime}, Z_{2}^{\prime \prime}\right)^{T}, \\
& \mathbf{x}=\left(X_{1,1}, Y_{1,1}, X_{1,3}, Y_{1,3}, X_{2}, Y_{2}\right)^{T} .
\end{aligned}
$$

Section II B describes the inversion of this operator for a given vector of macroparameters $\mathbf{f}_{0}$. We write the solution of the problem formally in the operator form

$$
\mathbf{x}_{0}=\mathbf{A}_{0}^{-1}\left(\mathbf{f}_{0}\right),
$$

where $\mathbf{A}_{0}^{-1}$ is the inverse operator realized analytically as described in Sec. II B.

The general problem with self-fields included reads

$$
\mathbf{A}_{\mathbf{x}}(\mathbf{x})=\mathbf{f}_{0},
$$

where nonlinear operator $\mathbf{A}_{\mathbf{x}}(\cdot)$ is realized by a tracking procedure (see Sec. IV) for the given rf parameters vector $\mathbf{x}$. Let us note that the tracking operator depends on this vector.

We would like to use the analytical solution as a preconditioner at each iteration. Our experience shows that such an approach results in fast convergence (5-10 iterations). In order to derive the iteration scheme let us rewrite Eq. (36) in an equivalent form:

$$
\mathbf{x}=\mathbf{A}_{0}^{-1}\left[\mathbf{A}_{0}(\mathbf{x})+\mathbf{f}_{0}-\mathbf{A}_{\mathbf{x}}(\mathbf{x})\right] .
$$

From the last equation the iterative scheme,

$$
\begin{aligned}
& \mathbf{x}_{n}=\mathbf{A}_{0}^{-1}\left[\mathbf{A}_{0}\left(\mathbf{x}_{n-1}\right)+\mathbf{f}_{0}-\mathbf{A}_{\mathbf{x}}\left(\mathbf{x}_{n-1}\right)\right], \quad n>0, \\
& \mathbf{x}_{0}=\mathbf{A}_{0}^{-1}(\mathbf{f}),
\end{aligned}
$$

can be suggested. It can be rewritten in a more convenient form, where one iteration includes the following steps:

$$
\begin{aligned}
& \mathbf{f}_{n-1}=\mathbf{A}_{\mathbf{x}}\left(\mathbf{x}_{n-1}\right) \text { - doing the numerical tracking, } \\
& \Delta \mathbf{f}_{n-1}=\mathbf{f}_{0}-\mathbf{f}_{n-1} \text {-calculation of the residual in the macroscopic parameters, } \\
& \mathbf{g}_{n}=\mathbf{g}_{n-1}+\Delta \mathbf{f}_{n-1}, \mathbf{x}_{n}=\mathbf{A}_{0}^{-1}\left(\mathbf{g}_{n}\right) \text {-doing the analytical correction of the rf parameters. }
\end{aligned}
$$

This iterative procedure solves Eq. (36). The problem is solved when the vector of $\mathrm{rf}$ parameters $\mathbf{x}$ used in the numerical tracking realizes the desired compression scenario given by vector $\mathbf{f}_{0}$ (see Table I). In other words, we track the bunch through the bunch compressors and the accelerating modules with rf parameters found from the iterative procedure. Then we estimate the compression parameters: energy of the reference particle in the bunch compressors, compression ratios at the position of the reference particle, the first and second derivatives of the compression curve, etc. The problem is solved if the estimated parameters are close to the desired (given in Table I, for example).

The iterative scheme is robust and converges fast to the solution. We apply this iterative algorithm in the next section in order to find the working point for a two-stage bunch compressor system in FLASH. The results for a three-stage bunch compressor system in European XFEL and for lower charges in FLASH have been published recently in [15].

\section{MODELING OF TWO-STAGE BUNCH COMPRESSION IN FLASH FACILITY}

The Free-Electron Laser FLASH at DESY is the first user facility for VUV and soft X-ray laser like radiation using the SASE scheme. Since the summer of 2005, it provides coherent femtosecond light pulses to user experiments with impressive brilliance [1,26]. It includes two bunch compressors, a $\mathrm{C}$ chicane and an $\mathrm{S}$ chicane (see Fig. 4). These two chicanes have to compress the electron bunches to achieve the peak current of $2500 \mathrm{~A}$. After the recent upgrade in 2010, the third harmonic module was installed and the linearized bunch compression is now possible. In the following we describe a way to define a working point in the current technical constraints for a special case of bunch with charge of $1 \mathrm{nC}$. The results from tracking simulations will be presented as well.

\section{A. Definition of the working point}

Before looking for the rf parameters settings we have to define 12 macroparameters (see Sec. IIB, Appendix and Table I in Sec. I). These parameters define operator $\mathbf{A}_{0}$ and vector $\mathbf{f}_{0}$ in Eq. (34), which is an operator form of system (6).

The initial conditions $E_{0}, \delta_{0}^{\prime}, \delta_{0}^{\prime \prime}, \delta_{0}^{\prime \prime \prime}$ are obtained from numerical simulations of the rf gun with code ASTRA [22]. The code is used to model the self-consistent beam dynamics in the rf gun for the bunch with a charge of $1 \mathrm{nC}$. The initial energy after the $\operatorname{rf}$ gun $E_{0}$ is about $5 \mathrm{MeV}$. The current profile and the longitudinal phase space after the rf gun, before the booster $\mathbf{M}_{1,1}$, are shown in Fig. 1 .

The initial peak current after the gun is about $52 \mathrm{~A}$. Hence, in order to reach the peak current of $2.5 \mathrm{kA}$ we need the total compression given by

$$
C_{2} \equiv Z_{2}^{-1}=48 \text {. }
$$


After the recent upgrade the FLASH facility has the following technical constraints on the achieved voltages:

$V_{1,1} \leq 150 \mathrm{MV}, \quad V_{1,3} \leq 26 \mathrm{MV}, \quad V_{2} \leq 350 \mathrm{MV}$

The deflecting radii in the bunch compressors have to fulfill the restrictions

$$
1.4 \leq \frac{r_{1}}{\mathrm{~m}} \leq 1.93, \quad 5.3 \leq \frac{r_{2}}{\mathrm{~m}} \leq 16.8 .
$$

In order to correct the nonlinearity induced by the fundamental harmonic module $\mathrm{M}_{1,1}$ before compressor $\mathrm{BC}_{1}$, we need to use a deceleration in the third harmonic module $\mathrm{M}_{1,3}$. Hence, the nominal energies in $\mathrm{BC}_{1}$ and $\mathrm{BC}_{2}$ are fixed (with a safety margin of $5 \%$ left for the optimization and the self-fields compensation) as follows:

$$
\begin{aligned}
& E_{1}=0.95\left[E_{0}+\frac{8}{9} e \max V_{1,1}\right] \approx 130 \mathrm{MeV}, \\
& E_{2}=0.95\left(E_{1}+e \max V_{2}\right) \approx 450 \mathrm{MeV} .
\end{aligned}
$$

The factor $8 / 9$ in the first equation comes from the fact that the third harmonic cavity will decelerate the bunch by approximately $V_{1,3} \approx V_{1,1} / 9$ [8, Eq. (11)].

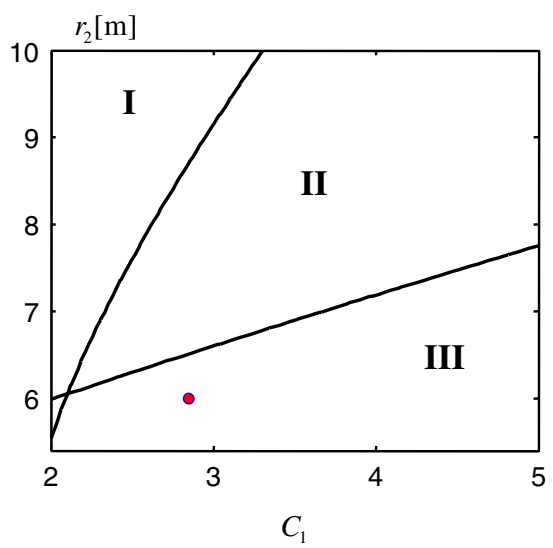

Now we are going to choose the deflecting radius $r_{1}$ in compressor $\mathrm{BC}_{1}$. In order to reduce the space-charge forces between the bunch compressors, we aim to use only a weak compression in $\mathrm{BC}_{1}$. Hence, the deflecting radius of the first bunch compressor is fixed at the maximum

$$
r_{1}=1.93 \mathrm{~m}
$$

This solution has two additional benefits: small CSR fields in compressor $\mathrm{BC}_{1}$ itself and a possibility of a larger energy chirp after it. The last feature reduces the voltage requirement on rf module $\mathrm{M}_{2}$.

Let us now choose the compression factor $C_{1} \equiv\left(Z_{1}\right)^{-1}$ in the first bunch compressor. We would like to take it as small as possible. For the time being, we will fix the free parameters of the global inverse compression at zero: $Z_{2}^{\prime}=0, \quad Z_{2}^{\prime \prime}=0$. From the analytical solution of Sec. II B (see the Appendix) we build the plots shown in Fig. 6. The left plot has three areas. In region I we need a very high voltage for the third harmonic module: $V_{1,3}>$ $26 \mathrm{MV}$. In region II we need a very high voltage for the second accelerating module: $V_{2}>360 \mathrm{MV}$. Hence, our

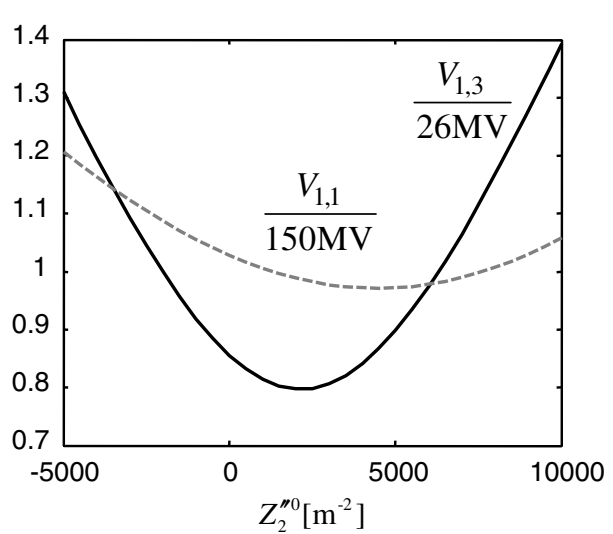

FIG. 6. The left plot shows the level lines for voltages. The circle presents the working point. The right plot shows the required voltages in module $M_{1}$ vs parameter $Z_{2}^{\prime \prime}$.
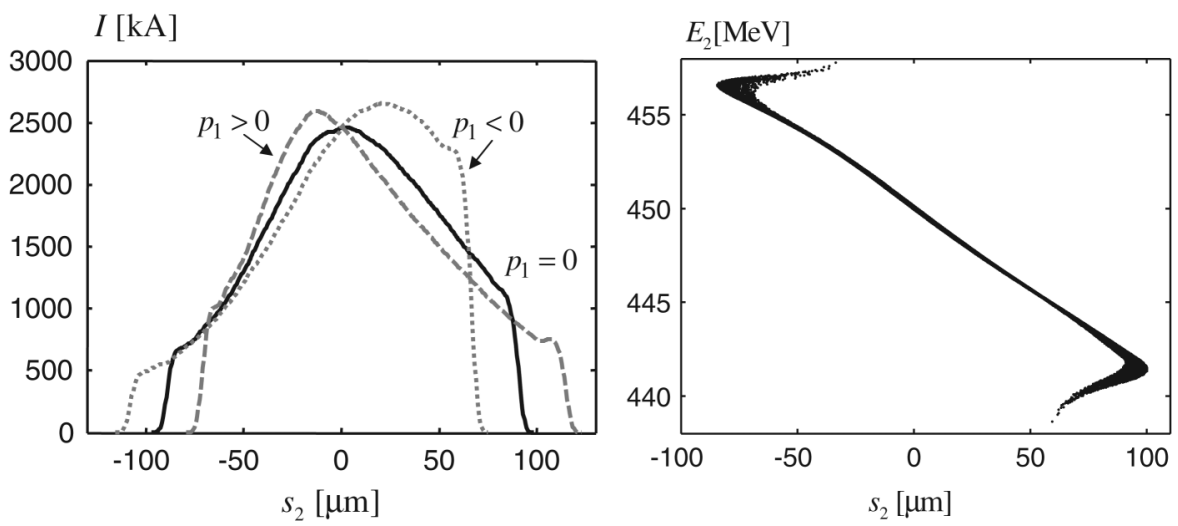

FIG. 7. Impact of the $Z_{2}^{\prime}$ parameter on the bunch shape (left plot). Longitudinal phase space after the second bunch compressor (right plot). 
TABLE II. The rf parameters in the working point.

\begin{tabular}{|c|c|c|c|c|c|c|}
\hline & $V_{1,1}, \mathrm{MV}$ & $\varphi_{1,1}$, degree & $V_{1,3}, \mathrm{MV}$ & $\varphi_{1,3}$, degree & $V_{2}, \mathrm{MV}$ & $\varphi_{2}$, degree \\
\hline Without self-fields & 148.49 & 10.52 & 21.02 & 180.77 & 345 & 21.95 \\
\hline With self-fields & 144.07 & -4.66 & 22.58 & 144.70 & 350.32 & 23.38 \\
\hline With increased wakefields & 217.89 & -51.85 & 68.02 & 97.48 & 358.6 & 26.44 \\
\hline
\end{tabular}

solution has to belong to region III. It can be seen from this plot that, due to the restriction on voltage $V_{1,3}$, the compression in the first $\mathrm{BC}$ cannot be less than 2. In order to have a reserve in $V_{1,3}$ for adjustment of global compression parameter $Z_{2}^{\prime \prime}$ and for the self-fields effects compensation, we choose

$$
C_{1}=2.84
$$

Now we are going to choose the deflecting radius $r_{2}$ in $\mathrm{S}$-chicane $\mathrm{BC}_{2}$. At the first step we will fix temporarily the phase $\varphi_{2}$ between the bunch compressors near to the maximum (with 10\% safety margin left for the optimization and the self-fields compensation)

$$
\varphi_{2}=0.9 \cos ^{-1}\left(\frac{E_{2}-E_{1}}{e \max \left(V_{2}\right)}\right) \approx 22^{\circ} .
$$

It means that we aim to produce the largest possible chirp with the rf system $\left(V_{2}, \varphi_{2}\right)$. At this case the energy chirp at the entrance of $\mathrm{BC}_{2}$ will be as large as only possible. Such a solution uses a larger deflecting radius $r_{2}$ in $\mathrm{BC}_{2}$. It results in weaker CSR fields in the last chicane.

In order to find the deflecting radius $r_{2}$ we have to solve the system

$$
\begin{aligned}
& \frac{1}{1-r_{561} \delta_{1}^{\prime}}=C_{1}, \quad \frac{1}{1-r_{562} \delta_{2}^{\prime} C_{1}}=\bar{C}_{2}, \\
& \delta_{2}^{\prime}=-e \frac{k}{C_{1}} \frac{Y_{2}}{E_{2}}+\frac{E_{1}}{E_{2}} \delta_{1}^{\prime}
\end{aligned}
$$

for unknown $\left(r_{562}, \delta_{1}^{\prime}\right)$. Here term $\bar{C}_{2}$ is the compression in compressor $\mathrm{BC}_{2}$ alone. The solution of this system reads

$$
\begin{aligned}
\delta_{1}^{\prime} & =\frac{(1+g)-\bar{C}_{2}^{-1}}{r_{561}(1+g)+E_{1}\left(E_{2}\right)^{-1} r_{562}}, \\
r_{562} & =\frac{\left(\bar{C}_{2}-1\right) r_{561}}{\bar{C}_{2}\left[\left(C_{1}-1\right) E_{1}\left(E_{2}\right)^{-1}-g\right]}, \quad g=k \frac{e Y_{2}}{E_{2}} r_{562} .
\end{aligned}
$$

Bunch compressor $\mathrm{BC}_{2}$ is of $\mathrm{S}$ type and the deflecting radius is given by [14]

$$
r_{2} \approx \frac{L_{B}}{\sin \sqrt{-r_{562} /\left(3 L_{B}+4 L_{D}\right)}}=6 \mathrm{~m},
$$

where $L_{B}=0.5$ is the magnet length and $L_{D}=0.5$ is the drift length between the magnets.

Equations (38)-(42) give six macroparameters from eight required to define system (6). We need now to choose values of $Z_{2}^{\prime}$ and $Z_{2}^{\prime \prime}$. As it was discussed already in Sec. II A we can avoid spikes in the current profile by choosing $Z_{2}^{\prime \prime}>0$.

In order to fix the positive value of the parameter $Z_{2}^{\prime \prime}$, we consider the right plot shown in Fig. 6. It presents the required voltages in module $M_{1}$ vs parameter $Z_{2}^{\prime \prime}$. In order to minimize the requirements on the voltages in this module, we choose

$$
Z_{2}^{\prime \prime}=2000 \mathrm{~m}^{-2} \text {. }
$$

Finally, we would like to fix the last parameter $Z_{2}^{\prime}$. With the help of this parameter we can shift the maximum of the compression to the right or to the left as shown in Fig. 7. We fix it at

$$
Z_{2}^{\prime}=1 \mathrm{~m}^{-1}
$$

to symmetrize the current profile.
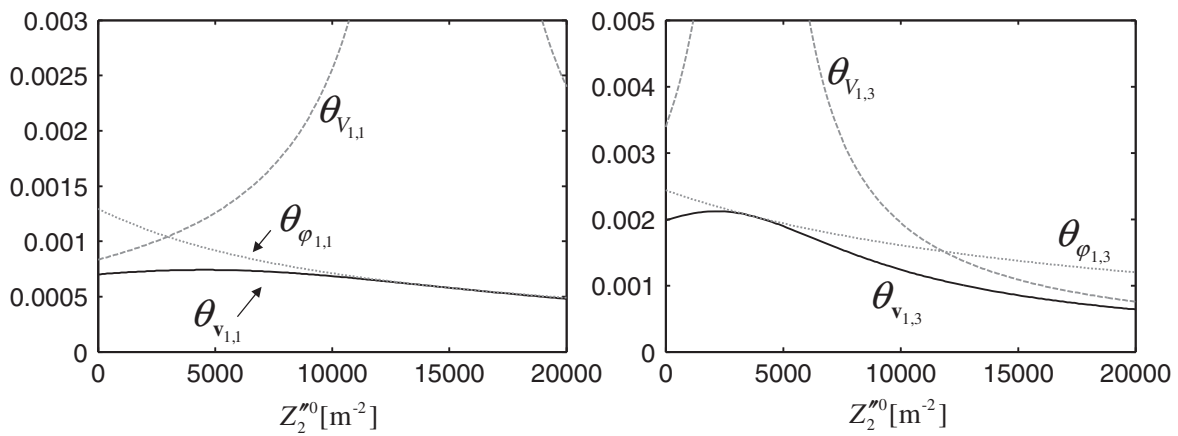

FIG. 8. The rf tolerances in accelerating module $M_{1}$ vs global compression parameter $Z_{2}^{\prime \prime 0}$. 
TABLE III. The rf tolerances in the working point.

\begin{tabular}{lccc}
\hline \hline & $M_{1,1}$ & $M_{1,3}$ & $M_{2}$ \\
\hline$\theta_{\varphi}$ & 0.00111 & 0.0022 & 0.0026 \\
$\theta_{V}$ & 0.00096 & 0.0075 & 0.0042 \\
$\theta_{\mathbf{v}_{1,1}}$ & 0.00072 & 0.0021 & 0.0022 \\
\hline \hline
\end{tabular}

Equations (38)-(44) define completely system (6) and from the analytical solution of Sec. II B we can find the rf parameters given in Table II (the first row).

Let us estimate tolerances for relative change of compression $\Theta=\left|\Delta C_{2}\right| / C_{2}=0.1$. We use the analytical estimations of Sec. III C. The left plot in Fig. 8 presents the estimation of the relative voltage and phase deviations admissible in module $\mathrm{M}_{1,1}$. These tolerances are obtained from Eqs. (21)-(26). By the solid line we show the strongest tolerance in two-dimensional space $\left(X_{1,1}, Y_{1,1}\right)$. It is given by Eq. (27). The same tolerances are shown for the third harmonic module at the right plot in Fig. 8. Table III presents all $\mathrm{rf}$ tolerances for the working point defined in this section.

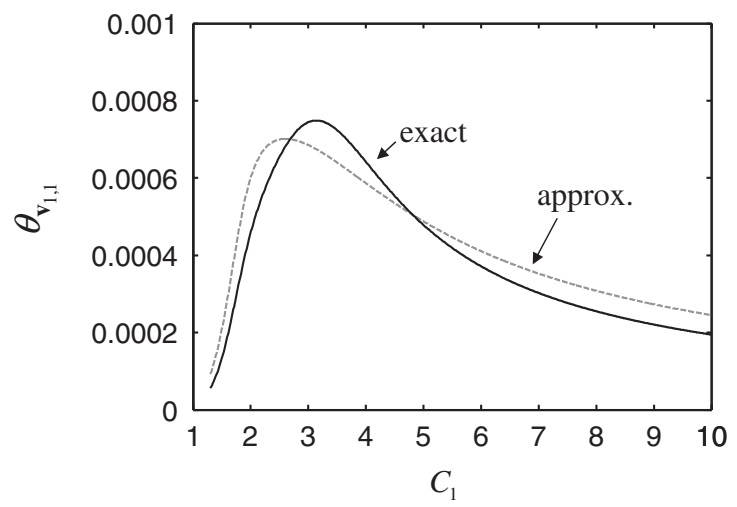

FIG. 9. The rf tolerance in accelerating module $\mathrm{M}_{1,1}$ vs compression in the first $\mathrm{BC}$.

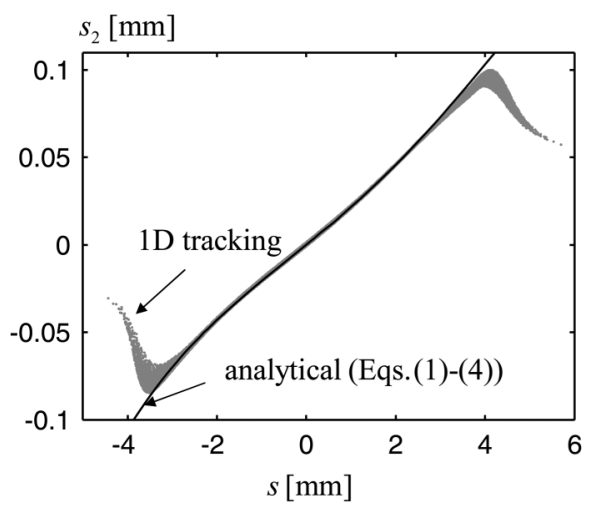

Finally, we show in Fig. 9 the dependence of the strongest tolerance in the booster $\mathrm{M}_{1,1}$ on the choice of the compression factor $C_{1}$ for the fixed factor $C_{2}=48$, and other parameters chosen as described above (see Table I). It is easy to see that the chosen value $C_{1}=2.84$ is near to the optimum. The solid curve presents the exact solution [Eq. (27)]. The approximate solution given by Eq. (28) is shown by the dashed line.

\section{B. Tracking simulations with collective effects}

In this section we present results for simulations with all collective effects included. We have implemented two different tracking procedures. The first procedure uses the analytical model of accelerating modules and tracks the transverse phase space by linear optics transform matrices. The longitudinal space-charge forces and wakefields are taken into account analytically as described in Sec. III A, Eqs. (31)-(33). The second procedure uses code ASTRA to track the particles through the accelerating sections of the beam line. The bunch compressors in both procedures are tracked with the help of code CSRTRACK. The first procedure is fast. It takes only about 10-20 min on one processor. The second procedure is very time consuming and takes hours of heavy parallelized calculations. We use the first model to implement the iterative procedure described in Sec. III B, Eq. (37). It takes about 5-10 iterations to solve the problem. After it we check the results with the full three-dimensional calculations implemented in the second procedure.

Figure 10 compares the results from the different tracking procedures to the analytical curve obtained from Eqs. (1)-(4). The coefficients of the third-order polynomial in Eq. (1) are estimated from the initial particle distribution presented in Fig. 1. As a first test we track the particles without collective effects with the rf parameters given in the first row of Table II. The result of this tracking coincides very well with the analytical curve (see the left plot in Fig. 10). Then we track the same particle distribution

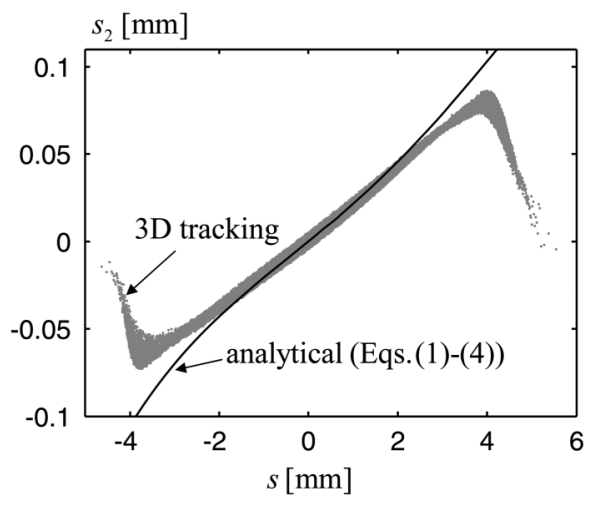

FIG. 10. The relative positions of the particles in the bunch after $\mathrm{BC}_{2}$ vs the initial positions after the gun. The left plot compares the analytical curve [Eqs. (1)-(4)] with the particle distribution from a simple one-dimensional tracking without collective effects. The right plot compares the analytical curve with the particle distribution from 3D self-consistent simulations. 

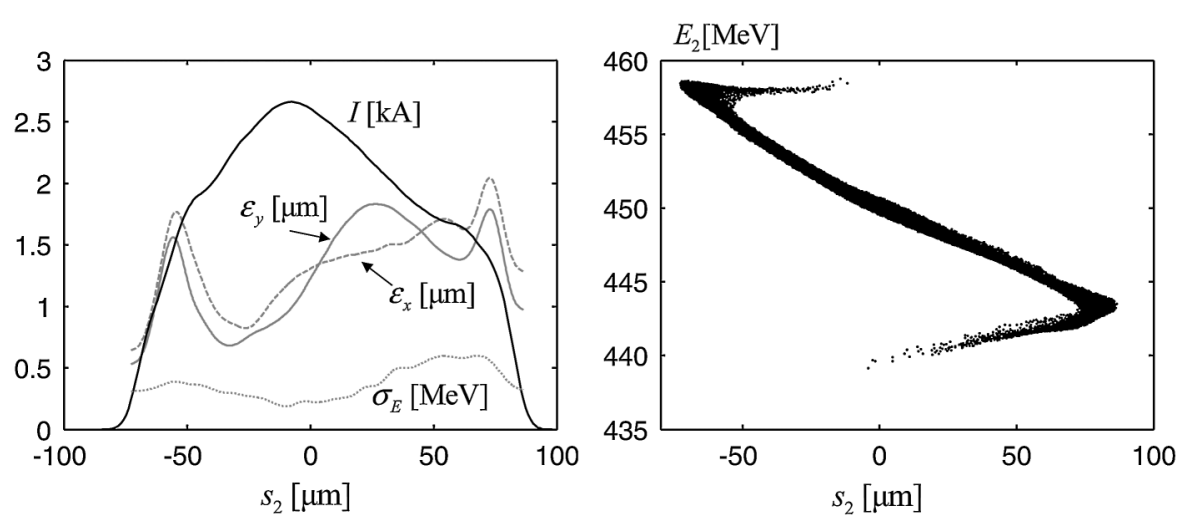

FIG. 11. The properties of the bunch after the second bunch compressor as obtained by 3D self-consistent simulations.

with all collective effects included. The rf parameters used in this procedure are given in the second row of Table II. The right plot in Fig. 10 compares the results of this threedimensional tracking with the analytical curve. We see a good agreement in the core of the bunch.

Figure 11 presents the properties of the bunch after the second bunch compressor as obtained with the full 3D modeling. The left plot shows current profile $I(s)$, horizontal slice emittance $\varepsilon_{x}(s)$, vertical slice emittance $\varepsilon_{y}(s)$, and rms slice energy spread $\sigma_{E}(s)$. The right plot presents the longitudinal phase space. It can be seen that the iterative procedure described in Sec. III B, Eq. (37), indeed has found the solution for the rf parameters which produces the desired longitudinal bunch compression. The found $\mathrm{rf}$ parameters are listed in the second row of Table II.

We have checked with the tracking that the tolerances are left approximately the same as described in Table III for the situation without self-fields.

\section{OTHER COMPRESSION SCENARIOS AND THE THEORY EXTENSIONS}

The analytical solution, presented in Sec. II, considers only nonlinearities in bunch compressors and rf fields. The method does not treat any collective effects. We hope that it is possible to include in the analytical solution (Sec. II B) the longitudinal wakefields and CSR effects at least in some simplified model (see, for example, [13]).

In our simulations presented in this paper, we take the collective effects into account by the tracking procedure described in Sec. III and correct the working point with a small number (5-10) of iterations. In order to show that the same procedure works and converges fast for the cases with stronger collective effects [as it has place in the Linac Coherent Light Source (LCLS) [2] and the Spring-8 Compact SASE Source [3]], we have repeated the simulations of Sec. IV with artificially increased longitudinal wakefields in rf modules. The rf frequency in LCLS is factor 2.2 higher than that used in FLASH. The longitudinal wakefields scale quadratically with the frequency [27]. Hence, we use the artificially increased by factor 5 longitudinal wakefields in our test simulation. Table II presents the working point for this case which was found with 10 iterations. We see a considerable shift in rf parameters, but the iterative numerical procedure itself produces the solution quite fast and reliable. This example confirms that the analytical solution of Sec. II cannot be applied straightforwardly to S-band and C-band structures used in LCLS [2]. It needs additional consideration to include the energy chirp due to the longitudinal wakefields.

Let us now analyze the compression scenario at LCLS reported in [28] from the point of view of rf tolerances in L0 linac (see [28] for layout). The working point has the following parameters: rf frequency $f=2.586 \mathrm{GHz}, \mathrm{BC}_{1}$ energy $E_{1}=250 \mathrm{MeV}, \mathrm{BC}_{2}$ energy $E_{2}=4.3 \mathrm{GeV}, \mathrm{BC}_{1}$ momentum compaction factor $r_{561}=-45.5 \mathrm{~mm}, \mathrm{BC}_{2}$ momentum compaction factor $r_{562}=-24.7 \mathrm{~mm}$, and total compression factor $C_{2}=90$. If we put these parameters in Eq. (30) then we obtain the optimal compression factor in $\mathrm{BC}_{1}$ equal to 5.7. In paper [28] a higher compression factor $C_{1}=7.6$ is used. In order to relax the rf tolerances and simultaneously to reduce the collective effects between the bunch compressors, a scenario with lower compression in $\mathrm{BC}_{1}$ is worth considering. However, it has to be checked with simulations including the strong wakefields existing in LCLS.

At the moment we continue our work on compression scenarios in the European XFEL. The preliminary results for a three-stage bunch compressor system in European XFEL and for lower charges in FLASH have been published recently in [15].

\section{SUMMARY}

In this paper we have derived an analytical solution for the multistage bunch compressor system with high harmonic module at the first stage. On the basis of this 
analytical solution, we have proposed an iterative procedure to find the working point from tracking simulations with collective effects included. The introduced formalism was applied to study the bunch compression in FLASH facility. The derivation of the analytical solution is quite general and can be generalized to more complicated configurations.

\section{ACKNOWLEDGMENTS}

We thank W. Decking and Ch. Behrens for useful discussions and corrections.

\section{APPENDIX A: EXPLICIT FORM OF THE SOLUTION FOR TWO AND THREE-STAGE BUNCH COMPRESSION SYSTEMS}

In this section we present the above derived analytical solution explicitly for two- and three-stage bunch compression schemes used or planned at [1-7].

In order to find six rf settings $\left(X_{1,1}, Y_{1,1}, X_{1,3}\right.$, $\left.Y_{1,3}, X_{2}, Y_{2}\right)$ in a two-stage bunch compression scheme, we have to define and to solve system (6) for $N=2$. To define six equations in system (6) we have to fix 12 independent parameters:

$$
\begin{aligned}
& E_{0}, \delta_{0}^{\prime}, \delta_{0}^{\prime \prime}, \delta_{0}^{\prime \prime \prime} \text {-initial conditions (as obtained from the gun simulations); } \\
& r_{1}, r_{2}, E_{1}, E_{2} \text {-deflecting radii and nominal energies in the bunch compressors; } \\
& Z_{1} \text {-inverse compression factor in bunch compressor } \mathrm{BC}_{1} \text {; } \\
& Z_{2}, Z_{2}^{\prime}, Z_{2}^{\prime \prime} \text { - parameters of the inverse global compression after } \mathrm{BC}_{2} \text {. }
\end{aligned}
$$

The solution of system (6) for the two-stage bunch compression system can be written explicitly:

$$
\begin{aligned}
& X_{2}=\bar{E}_{2}-\bar{E}_{1}, \quad \alpha_{1}=\frac{1-Z_{1}}{r_{561}}, \quad Y_{2}=\frac{\alpha_{1} \bar{E}_{1}-\delta_{2}^{\prime} \bar{E}_{2}}{k Z_{1}}, \quad \delta_{2}^{\prime}=\frac{Z_{1}-Z_{2}}{r_{562}}, \quad \alpha_{2}=\frac{y_{1}}{\bar{E}_{1}}, \quad y_{1}=\frac{Z_{2}^{\prime}-\tilde{x}_{2}}{\bar{x}_{2}}, \\
& \tilde{x}_{2}=\tilde{x}_{1}-\frac{r_{562}}{\bar{E}_{2}} \tilde{y}_{2}-2 t_{562}\left(\delta_{2}^{\prime}\right)^{2}, \quad \tilde{y}_{2}=-k^{2} Z_{1}^{2} X_{2}-k Y_{2} \tilde{x}_{1}, \quad \tilde{x}_{1}=-2 t_{561} \alpha_{1}^{2}, \quad \bar{x}_{2}=\bar{x}_{1}-\frac{r_{562}}{\bar{E}_{2}} \bar{y}_{2}, \\
& \bar{y}_{2}=1-k Y_{2} \bar{x}_{1}, \quad \bar{x}_{\Gamma}=-\frac{r_{561}}{\bar{E}_{1}}, \quad \alpha_{3}=\frac{\hat{y}_{1}}{\bar{E}_{1}}, \quad \hat{y}_{1}=\frac{Z_{2}^{\prime \prime} \hat{\tilde{x}}_{2}}{\bar{x}_{2}}, \quad \hat{\tilde{x}}_{2}=\hat{\tilde{x}}_{1}-\frac{r_{562}}{\bar{E}_{2}} \hat{\tilde{y}}_{2}-6 u_{562}\left(\delta_{2}^{\prime}\right)^{3}-6 t_{562} \delta_{2}^{\prime} \delta_{2}^{\prime \prime}, \\
& \delta_{2}^{\prime \prime}=\frac{\alpha_{2} \bar{E}_{1} \bar{y}_{2}+\tilde{y}_{2}}{\bar{E}_{2}}, \quad \hat{\tilde{y}}_{2}=k^{3} Z_{1}^{3} Y_{2}-3 k^{2} Z_{1} Z_{1}^{\prime} X_{2}-k Y_{2} \hat{\tilde{x}}_{1}, \quad \hat{\tilde{x}}_{1}=-6 u_{561} \alpha_{1}^{3}-6 t_{561} \alpha_{1} \alpha_{2}, \\
& Z_{1}^{\prime}=-r_{561} \alpha_{2}-2 t_{561} \alpha_{1}^{2} .
\end{aligned}
$$

The rf parameters $X_{1,1}, Y_{1,1}, X_{1,3}, Y_{1,3}$ can be found through relations (9).

In the case of a three-stage bunch compression scheme we have to define eight $\mathrm{rf}$ parameters $\left(X_{1,1}, Y_{1,1}, X_{1,3}, Y_{1,3}, X_{2}, Y_{2}, X_{3}, Y_{3}\right)$. In order to define eight equations in system (6), we have to fix 15 independent parameters:

$$
E_{0}, \delta_{0}^{\prime}, \delta_{0}^{\prime \prime}, \delta_{0}^{\prime \prime \prime} \text {-initial conditions (as obtained from the gun simulations); }
$$

$r_{1}, r_{2}, r_{3}, E_{1}, E_{2}, E_{3}$-deflecting radii and nominal energies in the bunch compressors;

$Z_{1}, Z_{2}$-inverse compression factors after compressor $\mathrm{BC}_{1}$ and after compressor $\mathrm{BC}_{2}$;

$$
Z_{3}, Z_{3}^{\prime}, Z_{3}^{\prime \prime} \text { - parameters of the inverse global compression after compressor } \mathrm{BC}_{3} \text {. }
$$

The solution for this configuration can be written explicitly: 


$$
\begin{aligned}
& X_{3}=\bar{E}_{3}-\bar{E}_{2}, \quad Y_{3}=\frac{\delta_{2}^{\prime} \bar{E}_{2}-\delta_{3}^{\prime} \bar{E}_{3}}{k Z_{2}}, \quad \delta_{3}^{\prime}=\frac{Z_{2}-Z_{3}}{r_{563}}, \quad \alpha_{2}=\frac{y_{1}}{\bar{E}_{1}}, \quad y_{1}=\frac{Z_{3}^{\prime}-\tilde{x}_{3}}{\bar{x}_{3}}, \\
& \tilde{x}_{3}=\tilde{x}_{2}-\frac{r_{563}}{\bar{E}_{3}} \tilde{y}_{3}-2 t_{563}\left(\delta_{3}^{\prime}\right)^{2}, \quad \tilde{y}_{3}=\tilde{y}_{2}-k^{2} Z_{2}^{2} X_{3}-k Y_{3} \tilde{x}_{2}, \quad \bar{x}_{3}=\bar{x}_{2}-\frac{r_{563}}{\bar{E}_{3}} \bar{y}_{3}, \quad \bar{y}_{3}=\bar{y}_{2}-k Y_{3} \bar{x}_{2}, \\
& \alpha_{3}=\frac{\hat{y}_{1}}{\bar{E}_{1}^{0}}, \quad \hat{y}_{1}=\frac{Z_{3}^{\prime \prime}-\hat{\tilde{x}}_{3}}{\bar{x}_{3}}, \quad \hat{\tilde{x}}_{3}=\hat{\tilde{x}}_{2}-\frac{r_{563}}{\bar{E}_{3}} \hat{\tilde{y}}_{3}-6 u_{563}\left(\delta_{3}^{\prime}\right)^{3}-6 t_{563} \delta_{3}^{\prime} \delta_{3}^{\prime \prime}, \quad \delta_{3}^{\prime \prime}=\frac{\alpha_{2} \bar{E}_{1} \bar{y}_{3}+\tilde{y}_{3}}{\bar{E}_{3}}, \\
& \hat{\tilde{y}}_{3}=\hat{\tilde{y}}_{2}+k^{3} Z_{2}^{3} Y_{3}-3 k^{2} Z_{2} Z_{2}^{\prime} X_{3}-k Y_{3} \hat{\tilde{x}}_{2}, \quad Z_{2}^{\prime}=Z_{1}^{\prime}-r_{562} \delta_{2}^{\prime \prime}-2 t_{562}\left(\delta_{2}^{\prime}\right)^{2} .
\end{aligned}
$$

Other rf parameters can be found by the same relation as for the two bunch compression system [see Eq. (A1)].

[1] W. Ackerman et al., Nat. Photon. 1, 336 (2007).

[2] J. Arthur et al., Report No. SLAC-R-593, 2002.

[3] SCSSX-FEL R\&D Group, SCSS X-FEL Conceptual Design Report, edited by T. Shintake and T. Tanaka (RIKEN Harima Institute/Spring-8, Sayo, Japan, 2004).

[4] M. Altarelli et al., Report No. DESY 2006-097, 2006.

[5] J. Amann et al., Report No. SLAC-R-930, 2009.

[6] R. Bartolini et al., in Proceedings of the 27th International Free Electron Laser Conference (JACoW, Liverpool, UK, 2009), p. 480.

[7] P. Tenenbaum, T. O. Raubenheimer, A. Wolski, in Proceedings of the 21st Particle Accelerator Conference, Knoxville, 2005 (IEEE, Piscataway, NJ, 2005), p. 2357.

[8] P. Emma, Technical Note No. LCLS-TN-01-1, 2001.

[9] T. I. Smith, Report No. SLAC-303, 1986.

[10] D. H. Dowell, T.D. Hayward, and A.M. Vetter, in Proceedings of the 1995 Particle Acceleration Conference, Dallas (IEEE, Piscataway, 1996), p. 992.

[11] K. Floettman, T. Limberg, and Ph. Piot, DESY Report No. TESLA-FEL 2001-06, 2001.

[12] K. Togawa, T. Hara, and H. Tanaka, Phys. Rev. ST Accel. Beams 12, 080706 (2009).

[13] R. A. Bosch, K. J. Kleman, and J. Wu, Phys. Rev. ST Accel. Beams 11, 090702 (2008).

[14] M. Dohlus, T. Limberg, and P. Emma, ICFA Beam Dynamics Newsletter 38, 15 (2005) [http://www-bd.fnal .gov/icfabd/Newsletter38.pdf].
[15] I. Zagorodnov, in Proceedings of the 32nd International Free Electron Laser Conference (JACoW, Malmo, Sweden, 2010), WEOBI2.

[16] M. Dohlus and T. Limberg, in Proceedings of the 27th International Free Electron Laser Conference (SLAC, Stanford, 2005), p. 250.

[17] I. Zagorodnov and M. Dohlus, Report No. DESY 10-102, 2010.

[18] M. Dohlus and T. Limberg, CSRTRACK Version 1.2 User's Manual, DESY, 2007.

[19] E. Saldin, E. Schneidmiller, and M. Yurkov, Nucl. Instrum. Methods Phys. Res., Sect. A 417, 158 (1998).

[20] M. Dohlus, A. Kabel, and T. Limberg, Nucl. Instrum. Methods Phys. Res., Sect. A 445, 338 (2000).

[21] G. Geloni, E. Saldin, and E. Schneidmiller, Nucl. Instrum. Methods Phys. Res., Sect. A 578, 34 (2007).

[22] K. Floettman, ASTRA Version 2.0 User's Manual, DESY, 2006.

[23] I. Zagorodnov and T. Weiland, Phys. Rev. ST Accel. Beams 8, 042001 (2005).

[24] T. Weiland and I. Zagorodnov, Report No. DESY TESLA03-23, 2003.

[25] I. Zagorodnov, T. Weiland, and M. Dohlus, DESY Report No. TESLA 2004-01, 2004.

[26] S. Schreiber, B. Faatz, and K. Honkavaara, in Proceedings of the 11th European Particle Accelerator Conference, Genoa, 2008 (EPS-AG, Genoa, Italy, 2008), p. 992.

[27] K. L. F. Bane, Report No. SLAC-PUB-4169, 1986.

[28] P. Emma, in Proceedings of the 23rd Particle Accelerator Conference, Vancouver, Canada, 2009 (IEEE, Piscataway, NJ, 2009), TH3PBI01. 